\title{
Multiple criteria hierarchy process for sorting problems based on ordinal regression with additive value functions
}

\author{
Salvatore Corrente $^{1} \cdot$ Michael Doumpos $^{2}$ - Salvatore Greco ${ }^{1,3}$. \\ Roman Słowiński $^{4,5}$. Constantin Zopounidis ${ }^{2,6}$
}

(C) Springer Science+Business Media New York 2015

\begin{abstract}
A hierarchical decomposition is a common approach for coping with complex decision problems involving multiple dimensions. Recently, the multiple criteria hierarchy process (MCHP) has been introduced as a new general framework for dealing with multiple criteria decision aiding in case of a hierarchical structure of the family of evaluation criteria. This study applies the MCHP framework to multiple criteria sorting problems and extends existing disaggregation and robust ordinal regression techniques that induce decision models from data. The new methodology allows the handling of preference information and the formulation of recommendations at the comprehensive level, as well as at all intermediate levels of the hierarchy of criteria. A case study on bank performance rating is used to illustrate the proposed methodology.
\end{abstract}

Michael Doumpos

mdoumpos@dpem.tuc.gr

Salvatore Corrente

salvatore.corrente@unict.it

Salvatore Greco

salgreco@unict.it

Roman Słowiński

roman.slowinski@cs.put.poznan.pl

Constantin Zopounidis

kostas@dpem.tuc.gr

1 Department of Economics and Business, University of Catania, Corso Italia, 55, 95129 Catania, Italy

2 School of Production Engineering and Management, Financial Engineering Laboratory, Technical University of Crete, University Campus, 73100 Chania, Greece

3 Portsmouth Business School, Centre of Operations Research and Logistics (CORL), University of Portsmouth, Richmond Building, Portland Street, Portsmouth PO1 3DE, UK

4 Institute of Computing Science, Poznań University of Technology, 60-965 Poznan, Poland

5 Systems Research Institute, Polish Academy of Sciences, 01-447 Warsaw, Poland

6 Audencia Nantes School of Management, Nantes, France 
Keywords Multiple criteria decision aiding $\cdot$ Multiple criteria hierarchy process $\cdot$ Sorting problems $\cdot$ Robust ordinal regression $\cdot$ Bank rating

\section{Introduction}

In many decision making problems, decisions concerning a set of alternatives are based on different evaluation criteria organized in a hierarchical structure. Such a hierarchy introduces a decomposition of the primary objective into separate dimensions, which are then further analyzed in sub-dimensions, up to the lowest level of the hierarchy, which consists of the elementary criteria. Structuring decision problems following such a hierarchical scheme is particularly useful in situations that require consideration of large sets of criteria describing different aspects of the problem at hand. Dealing with complex families of criteria of diverse nature, poses significant cognitive burden to decision makers (DMs). Thus, using a hierarchical decomposition facilitates the analysis as it allows DMs to deal with more manageable elementary dimensions. Furthermore, working with such a hierarchy provides detailed insights on all partial dimensions of the problem, instead of focusing solely on the comprehensive level.

A common approach to deal with hierarchies of criteria in MCDA is the analytic hierarchy process (Saaty 2005), but its fundamental problems are well-documented in the literature (see, for example, Bana e Costa and Vansnick 2008). Recently, the Multiple Criteria Hierarchy Process (MCHP) has been introduced as an alternative (Angilella et al. 2013; Corrente et al. 2012, 2013). The MCHP introduces a new modeling framework that allows the construction of sound decision models in decision problems with a hierarchical structure, through MCDA techniques based on the preference disaggregation paradigm (Jacquet-Lagrèze and Siskos 2001). The MCHP is able to take into account preference information not only at a comprehensive level but also at all lower levels of the hierarchy, and provide recommendations in a similar form.

In previous studies, the MCHP has been introduced in the context of choice and ranking problems, where the objective is either to choose the best alternative(s) among those considered (choice) or to rank-order the alternatives from the best to the worst ones. In these contexts, the MCHP has been employed to construct decision models with outranking methods such as ELECTRE and PROMETHEE (Corrente et al. 2013), value function models (Corrente et al. 2012), as well as with the Choquet integral preference model (Angilella et al. 2013).

In this study, we extend the MCHP framework to multiple criteria sorting (classification) problems, where the objective is to assign a set of alternatives to predefined (ordinal) decision classes. Such problems often arise in many domains (Zopounidis and Doumpos 2002) and they have attracted much interest in MCDA over the past decade. Sorting problems have been dealt in the literature using outranking relations (e.g., the ELECTRE Tri method Yu 1992), value functions, and decision rules (Greco et al. 1999, 2001, 2002, Słowiński et al. 2002). In this paper, we focus on value function models, which constitute a convenient and easy way of modeling DMs preferences in MCDA problems. The best-known method based on this modeling approach for multiple criteria sorting problems is the UTADIS method (UTilités Additives DIScriminantes) and its variants (Devaud et al. 1980; Doumpos and Zopounidis 2002; Zopounidis and Doumpos 1999). In this paper, we extend the UTADIS method to problems having a hierarchical structure by applying the MCHP framework. In order to reduce the cognitive effort of the DM, we also extend the UTADIS ${ }^{G M S}$ method (Greco et al. 2010) multiple putting it in the MCHP framework. UTADIS ${ }^{G M S}$ is the generalization of UTADIS to the Robust Ordinal Regression (ROR) setting (Corrente et al. 2013, 2014; 
Greco et al. 2008). ROR is a family of methods taking into account not only one but all instances of an assumed preference model being compatible with the preference information provided by the DM. In that regard, this study contributes to the literature on multiple criteria sorting through the extension of existing techniques for inferring decision models from sorting decision examples, using a formal framework of MCHP, which allows the input preference information to be decomposed into smaller and more manageable aspects of the problem. In order to illustrate the proposed methodology, we employ a case study involving a financial decision problem, namely the performance rating of banks. In a supervisory context, bank rating is a complex process that requires the consideration of all aspects of bank operation, financial status, and risk profile. This case study fits well the framework of MCHP and multiple criteria sorting, and thus, it illustrates well the potentials of the proposed modeling approach in practice.

The rest of the paper is organized as follows: In the next section, a general problem setting is provided. Section 3 describes the MCHP extension of the UTADIS method to decision problems with a hierarchical structure, while in Sect. 4 the integration of MCHP and UTADIS ${ }^{G M S}$ is explained in detail. The application to bank performance evaluation is presented in Sect. 5. Finally, Sect. 6 concludes the paper and provides some future research directions.

\section{General setting}

A set of alternatives $A=\{a, b, \ldots\}$ is evaluated on a set of criteria structured in a hierarchical way in $l$ different levels. The complete set of criteria (from all levels) will be denoted by $\mathcal{G}$, while the set of indices of criteria will be denoted by $\mathcal{I}_{\mathcal{G}}$. The criteria at the lowest level of the hierarchy will be called elementary criteria and the alternatives will be directly evaluated on these criteria only. The set of indices of elementary criteria will be denoted by $E L$, while the set of indices of elementary criteria descending from node $G_{\mathbf{r}}$ of the hierarchy $\left(\mathbf{r} \in \mathcal{I}_{\mathcal{G}}\right)$, will be denoted by $E\left(G_{\mathbf{r}}\right)$. Each node of the hierarchy represents a particular sub-dimension of the problem, with $G_{0}$ corresponding to the root of the hierarchy (i.e., $G_{0}=\mathcal{G}$ ). Without loss of generality, we shall suppose that all elementary criteria are to be maximized (i.e., preference increases with the value of each criterion).

Furthermore, by $n(\mathbf{r})$ we shall denote the number of criteria $G_{(\mathbf{r}, 1)}, \ldots, G_{(\mathbf{r}, n(\mathbf{r}))}$ descending from $G_{\mathbf{r}}$ in the next (lower) level of the hierarchy. Obviously, the elementary criteria are not further decomposed into subcriteria. By $L B O$ we shall denote the indices of the criteria from the next-to-last level of the hierarchy, while $L B\left(G_{\mathbf{r}}\right)$ will denote the set of indices of criteria descending from $G_{\mathbf{r}}$ and located at the next-to-last level.

Assuming that the set of elementary criteria is mutually preferentially independent (Keeney and Raiffa 1993; Wakker 1989), their aggregation is possible with an additive value function $U: A \rightarrow[0,1]$, such that:

$$
U(a)=\sum_{\mathbf{t} \in E L} u_{\mathbf{t}}\left(g_{\mathbf{t}}(a)\right)
$$

where $u_{\mathbf{t}}$ are marginal value functions related to elementary criteria $g_{\mathbf{t}}$.

In the MCHP context, assuming that at each level criteria are preferentially independent, it is possible to consider a partial value function for each (non-elementary) criterion $G_{\mathbf{r}}$, $\mathbf{r} \in \mathcal{I}_{\mathcal{G}}$ as follows: 


$$
U_{\mathbf{r}}(a)=\sum_{\mathbf{t} \in E\left(G_{\mathbf{r}}\right)} u_{\mathbf{t}}\left(g_{\mathbf{t}}(a)\right) .
$$

An obvious consequence is that

$$
U_{\mathbf{r}}(a)=\sum_{j=1}^{n(\mathbf{r})} U_{(\mathbf{r}, j)}(a)
$$

where $U_{(\mathbf{r}, j)}(a)$ represents the value of alternative $a$ according to the $j$ th subcriterion of $G_{\mathbf{r}}$, for all $\mathbf{r} \in \mathcal{I}_{\mathcal{G}} \backslash E L$ (more details on MCHP can be found in Corrente et al. 2012).

For each criterion $G_{\mathbf{r}}$ above the level of elementary criteria, the sorting procedure with respect to subcriteria descending directly from $G_{\mathbf{r}}$ consists in assigning each alternative from $A$ to one among $p_{\mathbf{r}}$ decision classes $C_{1}, \ldots, C_{p_{\mathbf{r}}}$, where $C_{p_{\mathbf{r}}}$ is the class of top performing alternatives and $C_{1}$ is the class of the worst alternatives. Note that the sorting with respect to subcriteria descending directly from different criteria $G_{\mathbf{r}}$ could involve different values of $p_{\mathbf{r}}$, i.e., the number of classes to which an alternative can be assigned could depend on $G_{\mathbf{r}}$. For each criterion $G_{\mathbf{r}}$ above the elementary level, class $C_{h}\left(h \in\left\{1, \ldots, p_{\mathbf{r}}\right\}\right)$ is defined by lower and upper value thresholds $b_{h-1}^{\mathbf{r}}$ and $b_{h}^{\mathbf{r}}$, such that $b_{h-1}^{\mathbf{r}}<b_{h}^{\mathbf{r}}$, defined on the value function scale. It follows that $0=b_{0}^{\mathbf{r}}<b_{1}^{\mathbf{r}}<\ldots<b_{p_{\mathbf{r}}-1}^{\mathbf{r}}<b_{p_{\mathbf{r}}}^{\mathbf{r}}=\sum_{\mathbf{t} \in E\left(G_{\mathbf{r}}\right)} u_{\mathbf{t}}\left(x_{\mathbf{t}}^{m_{\mathbf{t}}}\right)$, where the value of $b_{p_{\mathbf{r}}}^{\mathbf{r}}$ is the maximum level of the value function for $G_{\mathbf{r}}$ (with $x_{\mathbf{t}}^{m_{\mathbf{t}}}$ being the best performance on elementary criterion $g_{\mathbf{t}}$ over all alternatives from $A$ ).

\section{MCHP and the UTADIS method}

Consider an assignment of alternative $a \in A$ to class $C_{h}\left(h \in\left\{1, \ldots, p_{\mathbf{r}}\right\}\right)$ with respect to subcriteria descending directly from criterion $G_{\mathbf{r}}\left(\mathbf{r} \in \mathcal{I}_{\mathcal{G}} \backslash E L\right)$. In the following, instead of criterion $G_{\mathbf{r}}$, we shall often use the term node $G_{\mathbf{r}}$, in order to stress that the assignment takes place in a particular place of the hierarchy tree.

Moreover, the assignment of alternatives with respect to subcriteria descending directly from criterion $G_{\mathbf{r}}\left(\mathbf{r} \in \mathcal{I}_{\mathcal{G}} \backslash E L\right)$ will be called the assignment in node $G_{\mathbf{r}}$.

Definition 3.1 In node $G_{\mathbf{r}}\left(\mathbf{r} \in \mathcal{I}_{\mathcal{G}} \backslash E L\right)$, alternative $a$ is assigned to class $C_{h}$ ( $h=$ $\left.1, \ldots, p_{\mathbf{r}}\right)\left(\right.$ denoted as $\left.a \underset{\mathbf{r}}{\rightarrow} C_{h}\right)$, iff $b_{h-1}^{\mathbf{r}} \leq U_{\mathbf{r}}(a)<b_{h}^{\mathbf{r}}$.

As a consequence, in node $G_{\mathbf{r}}$,

- $a$ is assigned to at least class $C_{h}\left(a \underset{\mathbf{r}}{\rightarrow} C_{\geq h}\right)$, iff $U_{\mathbf{r}}(a) \geq b_{h-1}^{\mathbf{r}}$,

- $a$ is assigned to at most class $C_{h}\left(a \underset{\mathbf{r}}{\rightarrow} C_{\leq h}\right)$, iff $U_{\mathbf{r}}(a)<b_{h}^{\mathbf{r}}$ (the inequality becomes weak if $h=p_{\mathbf{r}}$, that is $\left.U_{\mathbf{r}}(a) \leq b_{p_{\mathbf{r}}}^{\mathbf{r}}\right)$,

- $a$ is assigned to some class in the interval $\left[C_{h_{1}}, C_{h_{2}}\right]\left(1<h_{1}<h_{2}<p_{\mathbf{r}}\right)$ $\left(a \underset{\mathbf{r}}{\rightarrow}\left[C_{h_{1}}, C_{h_{2}}\right]\right)$, iff $b_{h_{1}-1}^{\mathbf{r}} \leq U_{\mathbf{r}}(a)<b_{h_{2}}^{\mathbf{r}}$.

In what follows, in order to simplify the presentation and without loss of generality, we assume that the same classes apply in all nodes of the hierarchy tree. This means that the number of classes $p_{\mathbf{r}}$ to which each alternative can be assigned does not depend on the considered node $G_{\mathbf{r}}$. Consequently, $p_{\mathbf{r}}=p$ for all $\mathbf{r} \in \mathcal{I}_{\mathcal{G}} \backslash E L$.

A first desirable coherence property for hierarchical multiple criteria sorting methods is the following. If an alternative $a \in A$ is assigned to class $C_{h}$ in all nodes directly descending 
from $G_{\mathbf{r}}$, then it should also be assigned to the same class in node $G_{\mathbf{r}}$. For example, if student $S$ is assigned to the class of good students in the nodes corresponding to Algebra and Analysis, being the only two subcriteria of the criterion Mathematics, then $S$ has to be assigned to the class of good students also in the node of Mathematics. Henceforth, we shall refer to this property as the first coherence property of hierarchical multiple criteria sorting.

A second desirable coherence property for hierarchical multiple criteria sorting methods is the following. If an alternative $a \in A$ is assigned to at least class $C_{h}$, i.e., to class $C_{h}$ or better, in all nodes directly descending from $G_{\mathbf{r}}$, then it should also be assigned to at least class $C_{h}$ in node $G_{\mathbf{r}}$. Coming back to the previous example, if student $S$ is assigned to at least medium class of students in the nodes corresponding to Algebra and Analysis, then $S$ has also to be assigned to at least the medium class of students in the node of Mathematics. Let us call this property second coherence property of hierarchical multiple criteria sorting. Of course, another coherence property for hierarchical multiple criteria sorting methods is symmetric to the second property, i.e., if an alternative $a \in A$ is assigned to at most class $C_{h}$ (to class $C_{h}$ or worse), in all nodes directly descending from $G_{\mathbf{r}}$, then it should also be assigned to at most class $C_{h}$ in node $G_{\mathbf{r}}$. Henceforth, this property will be referred to as the third coherence property of hierarchical multiple criteria sorting. The second and third coherence properties of hierarchical multiple criteria sorting can be synthesized as follows: in node $G_{\mathbf{r}}$, an alternative $a \in A$ should be assigned to an interval of contiguous classes, included in the interval of classes having as extrema the worst and the best classes to which $a$ is assigned in nodes directly descending from $G_{\mathbf{r}}$. For example, if student $S$ is assigned to the interval of classes from moderate to relatively good students in the node of Algebra, and to the interval of classes from medium to good students in the node of Analysis, then student $S$ has to be assigned to an interval of classes from moderate to good students in the node of Mathematics. Even if this coherence property is the mere synthesis of the above second and third coherence properties, we shall refer to it as the fourth coherence property for hierarchical multiple criteria sorting methods, because it will be useful to recall it in the subsequent discussion.

Proposition 3.1 given below says that the first and the fourth coherence properties for hierarchical multiple criteria sorting methods coincide, and that they hold if and only if the value thresholds separating the classes in node $G_{\mathbf{r}}$ are equal to the sum of the corresponding value thresholds separating the classes in the nodes directly descending from $G_{\mathbf{r}}$ (see the Appendix for the proofs). Indeed, this condition is expressed as statement 1 of Proposition 3.1 , whereas the first and the fourth coherence properties for hierarchical multiple criteria sorting methods correspond to statements 2 and 3, respectively.

Proposition 3.1 The three following statements are equivalent:

1. In each node $G_{\mathbf{r}}, \mathbf{r} \in \mathcal{I}_{\mathcal{G}} \backslash E L, b_{h}^{\mathbf{r}}=\sum_{j=1}^{n(\mathbf{r})} b_{h}^{(\mathbf{r}, j)}$ for all $h=0, \ldots, p$,

2. In each node $G_{\mathbf{r}}, \mathbf{r} \in \mathcal{I}_{\mathcal{G}} \backslash E L$, if a $\underset{(\mathbf{r}, j)}{\longrightarrow}\left[C_{h_{j}}, C_{k_{j}}\right]$ for all $j=1, \ldots, n(\mathbf{r})$, then $a \underset{\mathbf{r}}{\rightarrow}\left[C_{h}, C_{k}\right]$ where $h=\min _{j=1, \ldots, n(\mathbf{r})} h_{j}$, and $k=\max _{j=1, \ldots, n(\mathbf{r})} k_{j}$,

3. In each node $G_{\mathbf{r}}, \mathbf{r} \in \mathcal{I}_{\mathcal{G}} \backslash E L$, if a $\underset{(\mathbf{r}, j)}{\longrightarrow} C_{h}$ for all $j=1, \ldots, n(\mathbf{r})$ then $a \underset{\mathbf{r}}{\rightarrow} C_{h}$.

Since we would like our hierarchical sorting approach to respect points 2 and 3 of Proposition 3.1, we shall assume that $b_{h}^{\mathbf{r}}=\sum_{j=1}^{n(\mathbf{r})} b_{h}^{(\mathbf{r}, j)}$ in each node $G_{\mathbf{r}}, \mathbf{r} \in \mathcal{I}_{\mathcal{G}} \backslash E L$. As a consequence of this choice, it is sufficient to define the value thresholds in nodes from the next-to-last of 
the hierarchy, because for any other higher level node $G_{\mathbf{r}}, \mathbf{r} \in \mathcal{I}_{\mathcal{G}} \backslash\{E L \cup L B O\}$ it holds that

$$
b_{j}^{\mathbf{r}}=\sum_{\mathbf{s} \in L B\left(G_{\mathbf{r}}\right)} b_{j}^{\mathbf{s}}, \text { for all } j=0, \ldots, p .
$$

In order to construct the additive value function and define the value thresholds, one can use a direct or an indirect approach. In the former case, the DM is asked to specify explicitly the parameters of the model (value thresholds in this case), following a direct assessment protocol designed specifically for the type of model under consideration (for example of such a protocol for additive value models see Bouyssou and Marchant 2010). On the other hand, in an indirect approach (Jacquet-Lagrèze and Siskos 2001) the DM is asked to provide some comprehensive preference information on the assignment of some reference alternatives (i.e., taking into account the full set of criteria present in the hierarchy) and/or partial preference information (i.e., considering a particular dimension of the problem, corresponding to criterion $G_{\mathbf{r}}$, being an intermediate node in the hierarchy tree). With such preference information at hand, it is possible to infer values for the parameters of the model that are compatible with the judgments provided by the DM. This can be achieved considering the following set of constraints (in accordance with Greco et al. 2010, henceforth $(U, b)$ will be used to denote a value function and a set of value thresholds compatible with the preferences of the DM, whereas $\mathcal{U}$ will denote the set of all compatible instances of this model):

$$
\begin{aligned}
& \left.\begin{array}{l}
U_{\mathbf{r}}(a) \geq b_{h-1}^{\mathbf{r}}, \\
U_{\mathbf{r}}(a)-b_{h}^{\mathbf{r}} \leq-\varepsilon
\end{array}\right\} \text { if } a \underset{\mathbf{r}}{\rightarrow} C_{h} \\
& U_{\mathbf{r}}(a) \geq b_{h-1}^{\mathbf{r}} \text {, if } a \underset{\mathbf{r}}{\rightarrow} C_{\geq h} \\
& U_{\mathbf{r}}(a)-b_{h}^{\mathbf{r}} \leq-\varepsilon \text {, if } a \underset{\mathbf{r}}{\rightarrow} C_{\leq h} \\
& \left.\begin{array}{l}
U_{\mathbf{r}}(a) \geq b_{h_{1}-1}^{\mathbf{r}}, \\
U_{\mathbf{r}}(a)-b_{h_{2}}^{\mathbf{r}} \leq-\varepsilon
\end{array}\right\} \text { if } a \underset{\mathbf{r}}{\rightarrow}\left[C_{h_{1}}, C_{h_{2}}\right] \\
& u_{\mathbf{t}}\left(x_{\mathbf{t}}^{k}\right) \geq u_{\mathbf{t}}\left(x_{\mathbf{t}}^{k-1}\right), \quad k=1, \ldots, m_{\mathbf{t}}, \text { for all } \mathbf{t} \in E L, \\
& u_{\mathbf{t}}\left(x_{\mathbf{t}}^{0}\right)=0 \text {, for all } \mathbf{t} \in E L \text {, and } \sum_{\mathbf{t} \in E L} u_{\mathbf{t}}\left(x_{\mathbf{t}}^{m_{\mathbf{t}}}\right)=1 \\
& b_{h}^{\mathbf{s}} \geq b_{h-1}^{\mathbf{s}}+\varepsilon, h=1, \ldots, p \text {, for all } \mathbf{s} \in L B O \text {, } \\
& b_{0}^{\mathbf{s}}=0 \text {, and } b_{p}^{\mathbf{s}}=\sum_{\mathbf{t} \in E\left(G_{\mathbf{s}}\right)} u_{\mathbf{t}}\left(x_{\mathbf{t}}^{m_{\mathbf{t}}}\right) \text {, for all } \mathbf{s} \in L B O \text {, } \\
& b_{h}^{\mathbf{r}}=\sum_{\mathbf{s} \in L B\left(G_{\mathbf{r}}\right)} b_{h}^{\mathbf{s}} \text {, for all } h=0, \ldots, p \text {, and for all } \mathbf{r} \in \mathcal{I}_{\mathcal{G}} \backslash\{E L \cup L B O\}
\end{aligned}
$$

where $x_{\mathbf{t}}^{k}, k=0, \ldots, m_{\mathbf{t}}$, are the $m_{\mathbf{t}}+1$ different performances on elementary criterion $g_{\mathbf{t}}$ attained by alternatives in $A$ (arranged in ascending order); $x_{\mathbf{t}}^{0}$ and $x_{\mathbf{t}}^{m_{\mathbf{t}}}$ are, respectively, the worst and the best performances of alternatives on elementary criterion $g_{\mathbf{t}}$, while $\varepsilon$ is an auxiliary variable used to translate the strict inequality constraints to weak inequality constraints.

If $E^{A^{R}}$ is feasible and $\varepsilon^{*}>0$, where $\varepsilon^{*}=\max \varepsilon$ subject to $E^{A^{R}}$, then there exists at least one instance $(U, b)$ compatible with the preferences provided by the DM. The readers interested to conditions ensuring the existence of an additive representation of ordered partitions could look at Bouyssou and Marchant (2010).

Remark 3.1 Let us observe that if the number of classes considered in each node $G_{\mathbf{r}}$ is different (different values of $p_{\mathbf{r}}$ for all $G_{\mathbf{r}}$ ), then the direct and the indirect approaches explained above remain valid. In particular, in the indirect approach, one has to consider the 
set of constraints $E_{1}^{A^{R}}$ obtained from $E^{A^{R}}$ by replacing the last three constraints with the following ones:

$$
\begin{gathered}
b_{h}^{\mathbf{r}} \geq b_{h-1}^{\mathbf{r}}+\varepsilon, h=1, \ldots, p_{\mathbf{r}}, \quad \text { for all } \mathbf{r} \in \mathcal{I}_{\mathcal{G}} \backslash E L, \\
b_{0}^{\mathbf{r}}=0, \text { and } b_{p_{\mathbf{r}}}^{\mathbf{r}}=\sum_{\mathbf{t} \in E\left(G_{\mathbf{r}}\right)} u_{\mathbf{t}}\left(x_{\mathbf{t}}^{m_{\mathbf{t}}}\right), \text { for all } \mathbf{r} \in \mathcal{I}_{\mathcal{G}} \backslash E L .
\end{gathered}
$$

The last constraint in $E^{A^{R}}$ does not hold anymore [except for the case $h=0$ and $h=p_{\mathbf{r}}$ in consequence of Eq. (3)], since the number and meaning of the different thresholds obviously depend on the number of classes to which each alternative can be assigned in node $G_{\mathbf{r}}$. For example, let us consider a small hierarchy in which a root criterion $G_{\mathbf{0}}$ has subcriteria $G_{\mathbf{1}}$ and $G_{2}$, and each alternative can be assigned to two classes in node $G_{\mathbf{0}}\left(p_{\mathbf{0}}=2\right)$ while it can be assigned to three and four classes in nodes $G_{1}$ and $G_{\mathbf{2}}$, respectively $\left(p_{\mathbf{1}}=3\right.$ and $\left.p_{\mathbf{2}}=4\right)$. In this case, there will be three value thresholds in node $G_{\mathbf{0}}\left(\left\{b_{0}^{0}, b_{1}^{0}, b_{2}^{0}\right\}\right)$, four value thresholds in node $G_{\mathbf{1}}\left(\left\{b_{0}^{1}, b_{1}^{1}, b_{2}^{1}, b_{3}^{1}\right\}\right)$, and five value thresholds in node $G_{\mathbf{2}}\left(\left\{b_{0}^{2}, b_{1}^{2}, b_{2}^{2}, b_{3}^{2}, b_{4}^{2}\right\}\right)$.

\section{MCHP and the UTADIS ${ }^{G M S}$ method}

In general, more than one instance of the preference model could be compatible with the preference information provided by the DM. Each of these instances restores the given information in the same way, but each one of them could provide different recommendations on alternatives outside the reference set. In this case, choosing a single compatible instance of the preference model may lead to a loss of possibly important information. For this reason, Robust Ordinal Regression (ROR) (Corrente et al. 2013, 2014, Greco et al. 2008) takes into account the whole set of instances of the preference model compatible with the preference information provided by the DM, by building necessary and possible preference relations that hold for all or for at least one compatible instance of the preference model.

In the MCHP context, the DM could be therefore interested to know not only to which class an alternative could be necessarily or possibly assigned taking into account the whole set of criteria, but also to which class it could be necessarily or possibly assigned with respect to a criterion corresponding to a particular node of the hierarchy tree.

In this section, we extend UTADIS ${ }^{G M S}$ (Greco et al. 2010) to the MCHP context, by reformulating the definition of the necessary and possible assignments as follows:

Definition 4.1 In any node $G_{\mathbf{r}},\left(\mathbf{r} \in \mathcal{I}_{\mathcal{G}} \backslash E L\right)$ in the hierarchy tree,

- $a \in A$ is necessarily assigned to at least class $C_{h}$, denoted by $a \stackrel{N}{\underset{\mathbf{r}}{\longrightarrow}} C_{\geq h}$, iff $U_{\mathbf{r}}(a) \geq b_{h-1}^{\mathbf{r}}$ for all compatible $(U, b)$,

- $a \in A$ is possibly assigned to at least class $C_{h}$, denoted by $\underset{\mathbf{r}}{\stackrel{P}{\rightarrow}} C_{\geq h}$, iff $U_{\mathbf{r}}(a) \geq b_{h-1}^{\mathbf{r}}$ for at least one compatible $(U, b)$,

- $a \in A$ is necessarily assigned to at most class $C_{h}$, denoted by $a \underset{\mathbf{r}}{\stackrel{N}{\longrightarrow}} C_{\leq h}$, iff $U_{\mathbf{r}}(a)<b_{h}^{\mathbf{r}}$ for all compatible $(U, b)$,

- $a \in A$ is possibly assigned to at most class $C_{h}$, denoted by $a \underset{\mathbf{r}}{\stackrel{P}{\rightarrow}} C_{\leq h}$, iff $U_{\mathbf{r}}(a)<b_{h}^{\mathbf{r}}$ for at least one compatible $(U, b)$.

The above necessary and possible preference relations can be computed as follows: 
- $a \stackrel{N}{\underset{\mathbf{r}}{\rightarrow}} C_{\geq h}$ iff the set of constraints $E^{N}\left(a \underset{\mathbf{r}}{\rightarrow} C_{\geq h}\right)$ is infeasible or if $\varepsilon\left(a \underset{\mathbf{r}}{\stackrel{N}{\longrightarrow}} C_{\geq h}\right) \leq 0$, where $E^{N}\left(a \underset{\mathbf{r}}{\rightarrow} C_{\geq h}\right)=E^{A^{R}} \cup\left\{U_{\mathbf{r}}(a)+\varepsilon \leq b_{h-1}^{\mathbf{r}}\right\}$ and $\varepsilon\left(a \underset{\mathbf{r}}{\stackrel{N}{\rightarrow}} C_{\geq h}\right)=\max \varepsilon$, s.t. $E^{N}\left(a \underset{\mathbf{r}}{\rightarrow} C_{\geq h}\right)$;

- $a \underset{\mathbf{r}}{\stackrel{P}{\rightarrow}} C_{\geq h}$ iff the set of constraints $E^{P}\left(a \underset{\mathbf{r}}{\rightarrow} C_{\geq h}\right)$ is feasible and $\varepsilon\left(a \underset{\mathbf{r}}{\stackrel{P}{\longrightarrow}} C_{\geq h}\right)>0$, where $E^{P}\left(a \underset{\mathbf{r}}{\rightarrow} C_{\geq h}\right)=E^{A^{R}} \cup\left\{U_{\mathbf{r}}(a) \geq b_{h-1}^{\mathbf{r}}\right\}$ and $\varepsilon\left(a \underset{\mathbf{r}}{\stackrel{P}{\rightarrow}} C_{\geq h}\right)=\max \varepsilon$, s.t. $E^{P}\left(a \underset{\mathbf{r}}{\rightarrow} C_{\geq h}\right)$;

- $a \underset{\mathbf{r}}{\stackrel{N}{\rightarrow}} C_{\leq h}$ iff the set of constraints $E^{N}\left(a \underset{\mathbf{r}}{\rightarrow} C_{\leq h}\right)$ is infeasible or if $\varepsilon\left(a \underset{\mathbf{r}}{\stackrel{N}{\longrightarrow}} C_{\leq h}\right) \leq 0$, where $E^{N}\left(a \underset{\mathbf{r}}{\rightarrow} C_{\leq h}\right)=E^{A^{R}} \cup\left\{U_{\mathbf{r}}(a) \geq b_{h}^{\mathbf{r}}\right\}$ and $\varepsilon\left(a \stackrel{N}{\underset{\mathbf{r}}{\longrightarrow}} C_{\leq h}\right)=\max \varepsilon$, s.t. $E^{N}\left(a \underset{\mathbf{r}}{\rightarrow} C_{\leq h}\right)$;

- $a \underset{\mathbf{r}}{\stackrel{P}{\rightarrow}} C_{\leq h}$ iff the set of constraints $E^{P}\left(a \underset{\mathbf{r}}{\rightarrow} C_{\leq h}\right)$ is feasible and $\varepsilon\left(a \underset{\mathbf{r}}{\stackrel{P}{\rightarrow}} C_{\leq h}\right)>0$, where $E^{P}\left(a \underset{\mathbf{r}}{\rightarrow} C_{\leq h}\right)=E^{A^{R}} \cup\left\{U_{\mathbf{r}}(a)+\varepsilon \leq b_{h}^{\mathbf{r}}\right\}$ and $\varepsilon\left(a \stackrel{P}{\rightarrow} C_{\leq h}\right)=\max \varepsilon$, s.t. $E^{P}\left(a \underset{\mathbf{r}}{\rightarrow} C_{\leq h}\right)$.

Robust hierarchical multiple criteria sorting methods should satisfy some desirable properties. The first two properties are logical properties, that, in fact, have to be satisfied even when there is no hierarchical structure. These properties state that for all $a \in A$ and for any non-elementary criterion $G_{\mathbf{r}}$,

1R) either $a$ is necessarily assigned to at least class $C_{h}$, or $a$ is possibly assigned to at most class $C_{h-1}, h \in\{2, \ldots, p\}$,

2R) either $a$ is necessarily assigned to at most class $C_{k}$, or $a$ is possibly assigned to at least class $C_{k+1}, k \in\{1, \ldots, p-1\}$.

Conditions $1 \mathbf{R}$ ) and $2 \mathbf{R}$ ) can be considered as completeness properties for robust hierarchical multiple criteria sorting corresponding to completeness properties for "flat" (non-hierarchical) sorting problems, according to which for all $a \in A$ :

1B) either $a$ is assigned to at least class $C_{h}$, or $a$ is assigned to at most class $C_{h-1}$, $h \in\{2, \ldots, p\}$,

2B) either $a$ is assigned to at most class $C_{k}$, or $a$ is assigned to at least class $C_{k+1}$, $k \in\{1, \ldots, p-1\}$.

On the basis of this observation, we shall call properties $\mathbf{1 R}$ ) and $\mathbf{2 R}$ ), first and second completeness properties of robust hierarchical multiple criteria sorting. Observe that removing the reference to node $G_{\mathbf{r}}$ of the hierarchy tree, the first and the second completeness properties should hold for any robust multiple criteria sorting method.

Other desirable properties are related to the hierarchical nature of the robust sorting, and can be seen as counterparts of the robust multiple criteria sorting of the coherence properties considered in Sect. 3 for non-hierarchical multiple criteria sorting. The coherence properties for robust hierarchical multiple criteria sorting methods that we shall consider are the following:

- If $a$ is necessarily assigned to at least class $C_{h}$ in all nodes directly descending from $G_{\mathbf{r}}$, then it is necessarily assigned to at least class $C_{h}$ in node $G_{\mathbf{r}}$. For example, if student 
$S$ is necessarily assigned to at least class medium in both Algebra and Analysis, then $S$ has to be assigned to at least class medium also in the node of Mathematics. Let us call this property first coherence property for robust hierarchical multiple criteria sorting methods.

- If $a$ is necessarily assigned to at most class $C_{k}$ in all nodes directly descending from $G_{\mathbf{r}}$, then it is necessarily assigned to at most class $C_{k}$ in node $G_{\mathbf{r}}$. For example, if student $S$ is necessarily assigned to at most class moderate in both Algebra and Analysis, then $S$ has to be assigned to at most class moderate also in the node of Mathematics. Let us call this property second coherence property for robust hierarchical multiple criteria sorting methods.

- If $a$ is necessarily assigned to at least class $C_{h}$ in all nodes directly descending from $G_{\mathbf{r}}$, with the possible exception of node $\bar{j}$ for which $a$ is possibly assigned to at least class $C_{h}$, then $a$ is possibly assigned to at least class $C_{h}$ in node $G_{\mathbf{r}}$. For example, if student $S$ is assigned to at least class medium necessarily in the node of Algebra, and possibly in the node of Analysis, then $S$ has to be possibly assigned to at least class medium also in the node of Mathematics. Let us call this property third coherence property for robust hierarchical multiple criteria sorting methods.

- If $a$ is necessarily assigned to at most class $C_{k}$ in all nodes directly descending from $G_{\mathbf{r}}$, with the possible exception of node $\bar{j}$ for which $a$ is possibly assigned to at most class $C_{k}$, then $a$ is possibly assigned to at most class $C_{k}$ in node $G_{\mathbf{r}}$. For example, if student $S$ is assigned to at most class moderate necessarily in the node of Algebra, and possibly in the node of Analysis, then $S$ has to be possibly assigned to at most class moderate also in the node of Mathematics. Let us call this property fourth coherence property for robust hierarchical multiple criteria sorting methods.

Proposition 4.1 given below says that the above two completeness properties, as well as the four coherence properties hold for the hierarchical UTADIS ${ }^{G M S}$ we are proposing. Notice that the four coherence properties are satisfied because statement 1 in Proposition 3.1 holds, i.e., because the value thresholds separating the classes in node $G_{\mathbf{r}}$ are equal to the sum of corresponding value thresholds separating the classes in all nodes directly descending from $G_{\mathbf{r}}$.

Proposition 4.1 In any node $G_{\mathbf{r}}, \mathbf{r} \in \mathcal{I}_{\mathcal{G}} \backslash E L$, of the hierarchy tree,

1. For all $a \in A$, and $h=2, \ldots$, p, either $a \underset{\mathbf{r}}{\stackrel{N}{\rightarrow}} C_{\geq h}$ or $\underset{\mathbf{r}}{\stackrel{P}{\rightarrow}} C_{\leq h-1}$,

2. For all $a \in A$, and $k=1, \ldots, p-1$, either $a \stackrel{N}{\rightarrow} C_{\leq k}$ or $\underset{\mathbf{r}}{\stackrel{P}{\rightarrow}} C_{\geq k+1}$,

3. If $a \underset{(\mathbf{r}, j)}{\stackrel{N}{\longrightarrow}} C_{\geq h_{j}}, j=1, \ldots, n(\mathbf{r})$, then $a \underset{\mathbf{r}}{\stackrel{N}{\longrightarrow}} C_{\geq h}$ where $h=\min _{j=1, \ldots, n(\mathbf{r})} h_{j}$,

4. If $a \underset{(\mathbf{r}, j)}{\stackrel{N}{\longrightarrow}} C_{\leq k_{j}}, j=1, \ldots, n(\mathbf{r})$, then $a \underset{\mathbf{r}}{\stackrel{N}{\longrightarrow}} C_{\leq k}$ where $k=\max _{j=1, \ldots, n(\mathbf{r})} k_{j}$,

5. If $a \underset{(\mathbf{r}, j)}{\stackrel{N}{\longrightarrow}} C_{\geq h_{j}}, j \in\{1, \ldots, n(\mathbf{r})\} \backslash\{\bar{j}\}$ and $a \underset{(\mathbf{r}, \vec{j})}{\stackrel{P}{\longrightarrow}} C_{\geq h_{\bar{j}}}$, then $a \underset{\mathbf{r}}{\stackrel{P}{\longrightarrow}} C_{\geq h}$ where $h=\min _{j=1, \ldots, n(\mathbf{r})} h_{j}$,

6. If $a \underset{(\mathbf{r}, j)}{\stackrel{N}{\longrightarrow}} C_{\leq k_{j}}, j \in\{1, \ldots, n(\mathbf{r})\} \backslash\{\bar{j}\}$, and $a \underset{(\mathbf{r}, \vec{j})}{\stackrel{P}{\longrightarrow}} C_{\leq k_{\bar{j}}}$ then $a \underset{\mathbf{r}}{\stackrel{P}{\longrightarrow}} C_{\leq k}$ where $k=\max _{j=1, \ldots, n(\mathbf{r})} k_{j}$.

An obvious consequence of Proposition 4.1 is that if $a \underset{(\mathbf{r}, j)}{\stackrel{N}{\longrightarrow}} C_{h}, j=1, \ldots, n(\mathbf{r})$, then $a \stackrel{N}{\longrightarrow} C_{h}$. 
In order to possibly or necessarily assign an alternative $a \in A$ to an interval of classes in node $G_{\mathbf{r}}$ of the hierarchy tree, the following indices can be defined:

$$
\begin{aligned}
& L_{\mathbf{r}}^{\mathcal{U}, P}(a)=\max \left(\{1\} \cup\left\{h \in H: a \underset{\mathbf{r}}{\stackrel{N}{\rightarrow}} C_{\geq h}\right\}\right), \\
& R_{\mathbf{r}}^{\mathcal{U}, P}(a)=\min \left(\{p\} \cup\left\{h \in H: a \underset{\mathbf{r}}{\stackrel{N}{\rightarrow}} C_{\leq h}\right\}\right) \\
& L_{\mathbf{r}}^{\mathcal{U}, N}(a)=\max \left(\{1\} \cup\left\{h \in H: a \underset{\mathbf{r}}{\stackrel{P}{\rightarrow}} C_{\geq h}\right\}\right), \\
& R_{\mathbf{r}}^{\mathcal{U}, N}(a)=\min \left(\{p\} \cup\left\{h \in H: a \underset{\mathbf{r}}{\stackrel{P}{\rightarrow}} C_{\leq h}\right\}\right) .
\end{aligned}
$$

On the basis of Proposition 4.1, we can prove the following results:

Proposition 4.2 In any node $G_{\mathbf{r}}\left(\mathbf{r} \in \mathcal{I}_{\mathcal{G}} \backslash E L\right)$ of the hierarchy tree, and $a \in A$,

1. $L_{\mathbf{r}}^{\mathcal{U}, P}(a) \geq \min _{j=1, \ldots, n(\mathbf{r})}\left\{L_{(\mathbf{r}, j)}^{\mathcal{U}, P}(a)\right\}$,

2. $R_{\mathbf{r}}^{\mathcal{U}, P}(a) \leq \max _{j=1, \ldots, n(\mathbf{r})}\left\{R_{(\mathbf{r}, j)}^{\mathcal{U}, P}(a)\right\}$.

\section{Application to bank performance rating}

\subsection{Problem context}

In order to illustrate the applicability of the proposed approaches, this section presents results from a case study involving bank performance rating, in a context of prudential supervision. Under the existing financial regulatory framework of Basel II, the banking supervisory authorities of each country (e.g. central banks) should conduct performance assessments on a regular basis for banks operating in the country, in order to ensure the stability of the country's banking system. Given that bank defaults are rare events, adequate historical data are usually not available to fit statistical models for estimating the likelihood of financial distress for banking institutions. Therefore, supervisors mainly rely on judgmental peer assessment systems, which take into account all aspects of a bank's operations and risk profile (Comptroller of the Currency 2007; Sahajwala and Van den Bergh 2000; Greuning and Brajovic Bratanovic 2009). The application of the MCDA is well-suited in this context, as it provides bank analysts and supervisors with a formal framework and analytic techniques for constructing composite performance indicators, exploring the trade-offs between different risk and performance factors, conducting robustness checks, and exploring stress testing scenarios.

Typically, bank rating systems consider six major dimensions, which define a comprehensive assessment framework referred to as CAMELS:

(1) capital adequacy,

(2) asset quality,

(3) management competence,

(4) earning generating ability,

(5) liquidity,

(6) sensitivity to market risks.

These dimensions are further decomposed into elementary criteria, which are specified according to particular characteristics of the banking system in a country. Thus, the problem 


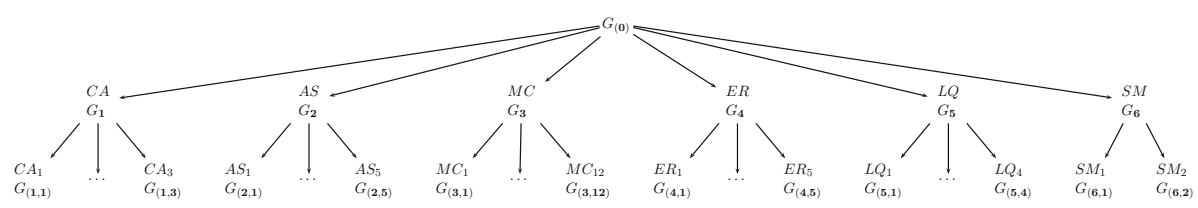

Fig. 1 Hierarchy of criteria

has a hierarchical structure and the bank rating assessment process should provide results not only at the comprehensive level, but also at each one of the above main dimensions. The results are commonly expressed in a 5-point rating scale. Thus, the context of bank rating fits well the MCHP sorting framework developed in this study.

\subsection{Data and criteria}

The data used in the analysis are taken from Doumpos and Zopounidis (2010) and they originate from the Bank of Greece (the supervisory authority responsible for the Greek banking system). They involve 18 Greek banks between 2001 and 2005 (overall 85 bank-year observations $^{1}$, which correspond to the alternatives). The banks have been evaluated on 31 criteria structured in a hierarchical way following the CAMELS framework, as shown in Fig. 1. The six CAMELS dimensions (Capital-CA, Assets-AS, Management-MC, EarningsER, Liquidity-LQ, and Sensitivity to market risks-SM) are the first level criteria, each analyzed through multiple subcriteria in the subsequent level. These subcriteria serve as the elementary decision attributes in the MCHP framework, for which the data are available for the banks in the sample.

The definition of the elementary criteria is given in Table 1 . These include 17 financial ratios that describe quantitative aspects of bank operation, whereas the remaining 14 criteria describe qualitative issues (but these are still measured on a $0.5-5.5$ cardinal scale defined by analysts at the Bank of Greece, with lower values indicating higher performance). Criteria whose type is indicated in Table 1 as "max" are positively related to the performance of banks, whereas "min" criteria are those that are negatively related to bank performance. For each elementary criterion $g_{\mathbf{t}}$, we considered a linear marginal value function:

$$
u_{\mathbf{t}}(a)=u_{\mathbf{t}}\left(x_{\mathbf{t}}^{m_{\mathbf{t}}}\right) \frac{g_{\mathbf{t}}(a)-x_{\mathbf{t}}^{0}}{x_{\mathbf{t}}^{m_{\mathbf{t}}}-x_{\mathbf{t}}^{0}}
$$

where the best $\left(x_{\mathbf{t}}^{m_{\mathbf{t}}}\right)$ and worst $\left(x_{\mathbf{t}}^{0}\right)$ performances are defined as follows:

$$
\begin{aligned}
& \text { Maximization criteria: } x_{\mathbf{t}}^{m_{\mathbf{t}}}=\max \left\{g_{\mathbf{t}}(a), a \in A\right\} \text { and } x_{\mathbf{t}}^{0}=\min \left\{g_{\mathbf{t}}(a), a \in A\right\} \\
& \text { Minimization criteria: } x_{\mathbf{t}}^{m_{\mathbf{t}}}=\min \left\{g_{\mathbf{t}}(a), a \in A\right\} \text { and } x_{\mathbf{t}}^{0}=\max \left\{g_{\mathbf{t}}(a), a \in A\right\} .
\end{aligned}
$$

It should be noted that the use of linear marginal value functions in the setting of this case study, is actually in accordance with the CAMELS modeling framework as implemented by the Bank of Greece. Furthermore, similar linear scoring and risk monitoring systems are widely used by bank supervisory agencies worldwide.

In accordance with the policy followed by analysts at the Bank of Greece during the period under consideration, the following points are taken into consideration:

1 For some banks the data were not available for all years.

The data are available at: http://www.fel.tuc.gr/BankData.xlsx 
Table 1 Evaluation criteria and their indices used in Fig. 1

\begin{tabular}{|c|c|c|c|c|c|}
\hline Category & Index & Abbr. & Type & Index & Criterion name \\
\hline \multirow[t]{3}{*}{ Capital } & \multirow[t]{3}{*}{1} & CA1 & $\operatorname{Max}$ & $(\mathbf{1}, 1)$ & Capital adequacy ratio \\
\hline & & CA2 & Min & $(\mathbf{1}, 2)$ & TIER II capital/TIER I \\
\hline & & CA3 & Min & $(\mathbf{1}, 3)$ & Qualitative* \\
\hline \multirow[t]{5}{*}{ Assets } & \multirow[t]{5}{*}{2} & AS1 & Min & $(2,1)$ & Risk-weighted assets/assets \\
\hline & & AS2 & Min & $(2,2)$ & (non performing loans - provisions)/Loans \\
\hline & & AS3 & Min & $(2,3)$ & Large exposures/(TIER I + TIER II capital) \\
\hline & & AS4 & Min & $(2,4)$ & $\begin{array}{l}\text { [0.5 (non performing loans) - } \\
\text { provisions }] \text { /equity }\end{array}$ \\
\hline & & AS5 & Min & $(2,5)$ & Qualitative* $^{*}$ \\
\hline \multirow[t]{12}{*}{ Management } & \multirow[t]{12}{*}{3} & MC1 & Min & $(3,1)$ & Operating expenses/operating income \\
\hline & & $\mathrm{MC} 2$ & Min & $(3,2)$ & Staff cost/assets \\
\hline & & MC3 & $\operatorname{Max}$ & $(3,3)$ & Operating income/business units \\
\hline & & $\mathrm{MC} 4$ & Min & $(3,4)$ & $\begin{array}{l}\text { Top management competencies, } \\
\text { qualifications and continuity }\end{array}$ \\
\hline & & MC5 & Min & $(3,5)$ & Managers' experience and competence \\
\hline & & MC6 & Min & $(3,6)$ & $\begin{array}{l}\text { Resilience to change, strategy, long term } \\
\text { horizon }\end{array}$ \\
\hline & & $\mathrm{MC7}$ & Min & $(3,7)$ & Management of information systems \\
\hline & & MC8 & Min & $(3,8)$ & Internal control systems \\
\hline & & MC9 & Min & $(3,9)$ & Financial risk management system \\
\hline & & $\mathrm{MC} 10$ & Min & $(3,10)$ & $\begin{array}{l}\text { Internal processes charter-implementation } \\
\text { monitoring }\end{array}$ \\
\hline & & MC11 & Min & $(3,11)$ & Timely and accurate data collection \\
\hline & & $\mathrm{MC} 12$ & Min & $(3,12)$ & Information technology systems \\
\hline \multirow[t]{5}{*}{ Earnings } & \multirow[t]{5}{*}{4} & ER1 & $\operatorname{Max}$ & $(4,1)$ & Net income/assets \\
\hline & & ER2 & $\operatorname{Max}$ & $(4,2)$ & Net income/equity \\
\hline & & ER3 & $\operatorname{Max}$ & $(4,3)$ & Interest revenue/assets \\
\hline & & ER4 & $\operatorname{Max}$ & $(4,4)$ & Other operating revenue/assets \\
\hline & & ER5 & Min & $(4,5)$ & Qualitative* \\
\hline \multirow[t]{4}{*}{ Liquidity } & \multirow[t]{4}{*}{5} & LQ1 & Max & $(5,1)$ & Cash/assets \\
\hline & & LQ2 & Min & $(5,2)$ & (loans - provisions)/deposits \\
\hline & & LQ3 & Min & $(5,3)$ & Real funding from credit institutions/assets \\
\hline & & LQ4 & Min & $(\mathbf{5}, 4)$ & Qualitative* \\
\hline \multirow[t]{2}{*}{ Market } & \multirow[t]{2}{*}{6} & SM1 & Min & $(6,1)$ & $\begin{array}{l}\text { Risk-weighted assets II/risk-weighted assets } \\
\text { (I \& II) }\end{array}$ \\
\hline & & SM2 & Min & $(6,2)$ & Qualitative* \\
\hline
\end{tabular}

* Undisclosed criteria related to qualitative aspects of the banks' operation

- The importance of quantitative criteria should be at least equal to $70 \%$. Even though criteria related to qualitative aspects of bank operation are particularly useful for describing important performance and risk factors in the medium-long term, they clearly entail some subjectivity on the way they are modeled and assessed. On the other hand, financial quantitative criteria, despite their shortcomings (e.g., potential manipulation of accounting reporting standards), are hard data widely used in prudential supervision research and 
practice all over the world. In that regard, this requirement is imposed to ensure that the resulting evaluation does not overweight the qualitative aspects of bank operation over the actual financial results.

Denoting by $\mathcal{I}_{\mathcal{G}_{\text {Qual }}}$ the set of indices of qualitative elementary criteria, that is

$$
\mathcal{I}_{\mathcal{G}_{\text {Qual }}}=\{(\mathbf{1}, 3),(\mathbf{2}, 5),(\mathbf{4}, 5),(\mathbf{5}, 4),(\mathbf{6}, 2)\}
$$

and by $\mathcal{I}_{\mathcal{G}_{\text {Quan }}}$ the set of indices of all quantitative elementary criteria $\left(\mathcal{I}_{\mathcal{G}_{\text {Qual }}} \cup \mathcal{I}_{\mathcal{G}_{\text {Quan }}}=\right.$ $\mathcal{I}_{\mathcal{G}}$ ), the previous piece of preference information can be translated to the following constraint:

$$
\sum_{\mathbf{t} \in \mathcal{I}_{\mathcal{G}_{\text {Quan }}}} u_{\mathbf{t}}\left(x_{\mathbf{t}}^{m_{\mathbf{t}}}\right) \geq 0.7 .
$$

This implies that a bank having the best performance on all quantitative elementary criteria should have a comprehensive value not less than 0.7 .

- Capital and assets are the most important dimensions, whereas market risk is the least important one. Capital adequacy and asset quality are critical factors for ensuring the financial soundness of a bank. They are both closely monitored on a regular basis by supervisors, and actions are taken whenever a bank does not have adequate capital (see for example the stress tests conducted by the European Banking Authority) or when its loan portfolio is particularly troublesome. Liquidity is also an important issue, but during the period of the analysis (2001-2005) there were no indications that liquidity risk could be a critical factor in the foreseeable future for Greek banks. Therefore, liquidity is considered to be of lower importance for this analysis, compared to capital adequacy and asset quality. The same applies to earning power and management competence, too. Earning power is an important dimension for the success of banking institutions as it indicates how they perform in multiple areas. Furthermore, a strong stream of earnings constitutes the first line of defense against loan losses. However, the period of the analysis was a time of transition for Greek banks in terms of their profitability, mainly due to the introduction of the Euro and the adoption of the international accounting standards by the largest banks. Due to the challenges that these issues created in assessing the earnings of Greek banking institutions over the period under consideration, its relative importance was set below capital and assets. On the other hand, management competence is mostly related to qualitative aspects of bank operation, which, as explained above, are given lower priority. Finally, the data set only involves commercial banks, whose exposure to market risks is limited. Therefore, the market risk dimension is assumed to be the least important one among the six criteria categories.

Using the notation introduced in Sect. 2 and indices of criteria shown in Table 1, the given three pieces of preference information can be translated to the following sets of constraints

$$
\begin{gathered}
\sum_{\mathbf{t} \in E\left(G_{\mathbf{1}}\right)} u_{\mathbf{t}}\left(x_{\mathbf{t}}^{m_{\mathbf{t}}}\right) \geq\left\{\begin{array}{l}
\sum_{\mathbf{t} \in E\left(G_{\mathbf{3}}\right)} u_{\mathbf{t}}\left(x_{\mathbf{t}}^{m_{\mathbf{t}}}\right)+\varepsilon, \\
\sum_{\mathbf{t} \in E\left(G_{\mathbf{4}}\right)} u_{\mathbf{t}}\left(x_{\mathbf{t}}^{m_{\mathbf{t}}}\right)+\varepsilon, \\
\sum_{\mathbf{t} \in E\left(G_{\mathbf{5}}\right)} u_{\mathbf{t}}\left(x_{\mathbf{t}}^{m_{\mathbf{t}}}\right)+\varepsilon, \\
\sum_{\mathbf{t} \in E\left(G_{\mathbf{6}}\right)} u_{\mathbf{t}}\left(x_{\mathbf{t}}^{m_{\mathbf{t}}}\right)+\varepsilon,
\end{array} \quad \sum_{\mathbf{t} \in E\left(G_{\mathbf{2}}\right)} u_{\mathbf{t}}\left(x_{\mathbf{t}}^{m_{\mathbf{t}}}\right) \geq\left\{\begin{array}{l}
\sum_{\mathbf{t} \in E\left(G_{\mathbf{3}}\right)} u_{\mathbf{t}}\left(x_{\mathbf{t}}^{m_{\mathbf{t}}}\right)+\varepsilon, \\
\sum_{\mathbf{t} \in E\left(G_{\mathbf{4}}\right)} u_{\mathbf{t}}\left(x_{\mathbf{t}}^{m_{\mathbf{t}}}\right)+\varepsilon, \\
\sum_{\mathbf{t} \in E\left(G_{\mathbf{5}}\right)} u_{\mathbf{t}}\left(x_{\mathbf{t}}^{m_{\mathbf{t}}}\right)+\varepsilon, \\
\sum_{\mathbf{t} \in E\left(G_{\mathbf{6}}\right)} u_{\mathbf{t}}\left(x_{\mathbf{t}}^{m_{\mathbf{t}}}\right)+\varepsilon,
\end{array}\right.\right. \\
\sum_{\mathbf{t} \in E\left(G_{\mathbf{6}}\right)} u_{\mathbf{t}}\left(x_{\mathbf{t}}^{m_{\mathbf{t}}}\right) \leq\left\{\begin{array}{l}
\sum_{\mathbf{t} \in E\left(G_{3}\right)} u_{\mathbf{t}}\left(x_{\mathbf{t}}^{m_{\mathbf{t}}}\right)-\varepsilon, \\
\sum_{\mathbf{t} \in E\left(G_{\mathbf{4}}\right)} u_{\mathbf{t}}\left(x_{\mathbf{t}}^{m_{\mathbf{t}}}\right)-\varepsilon, \\
\sum_{\mathbf{t} \in E\left(G_{\mathbf{5}}\right)} u_{\mathbf{t}}\left(x_{\mathbf{t}}^{m_{\mathbf{t}}}\right)-\varepsilon,
\end{array}\right.
\end{gathered}
$$


Table 2 Initial set of the expert's comprehensive judgments

\begin{tabular}{ll}
\hline Alternatives & Class \\
\hline$A_{16}, A_{17}, A_{18}$ & $C_{1}$ \\
$A_{10}, A_{21}, A_{22}$ & $C_{2}$ \\
$A_{7}, A_{8}, A_{9}$ & $C_{3}$ \\
$A_{1}, A_{2}, A_{6}$ & $C_{4}$ \\
$A_{3}, A_{4}, A_{5}$ & $C_{5}$ \\
\hline
\end{tabular}

where the constraints (8) say that criteria categories capital and assets are more important than the other four criteria categories, while the constraints (9) say that market risk is the least important criteria category. Let us notice that the constraints saying that market risk is less important than capital and assets are missing in (9) since these constraints are already present in (8).

\subsection{Discussion of results}

In addition to the above preference information, an expert banking analyst (DM) familiar with the Greek banking sector provided global assessments for a small set of banks, as shown in Table 2. These are banks for which the DM was familiar with their strengths and weaknesses over the examined period. For example, alternatives $A_{3}, A_{4}$, and $A_{5}$ correspond to a leading Greek bank in terms of its market niche and financial strength over a three years period (2003-2005), alternatives $A_{7}, A_{8}$, and $A_{9}$ involve a state-owned bank being in transition towards privatization, whereas $A_{16}, A_{17}$, and $A_{18}$ correspond to a recently privatized bank that faced significant operating challenges moving to a new corporate plan.

Since each bank can be assigned to one of five classes at the level of macro-criteria and at the comprehensive level, then six thresholds have to be specified for each macro-criterion $\left(b_{0}^{s}\right.$, $\left.b_{1}^{s}, b_{2}^{s}, b_{3}^{s}, b_{4}^{s}, b_{5}^{s}\right)$, such that $b_{0}^{s}=0$ and $b_{5}^{s}=\sum_{\mathbf{t} \in E\left(G_{s}\right)} u_{\mathbf{t}}\left(x_{\mathbf{t}}^{m_{\mathbf{t}}}\right)$ for all $s \in\{\mathbf{1}, \mathbf{2}, \mathbf{3}, \mathbf{4}, \mathbf{5}, \mathbf{6}\}$. Consequently, following Proposition 3.1, the thresholds for criterion $G_{0}$ are obtained as the sum of the corresponding thresholds for the six macro-criteria, that is $b_{h}^{0}=\sum_{s=1}^{6} b_{h}^{s}$, for all $h=0, \ldots, 5$.

Having defined the thresholds for the six macro-criteria, the preferences shown in Table 2 are translated to constraints as explained in Sect. 3. For example, the assignment at a comprehensive level of bank $A_{x}$ to class $C_{h}$ is translated to the constraints

$$
\left.\begin{array}{l}
U_{0}\left(A_{x}\right) \geq b_{h-1}^{0}, \\
U_{0}\left(A_{x}\right)-b_{h}^{0} \leq-\varepsilon .
\end{array}\right\}
$$

Consequently, the set $E^{A^{R}}$ containing the constraints translating the preferences of the DM and the technical constraints will be the following:

(7) $-(10)$,

$u_{\mathbf{t}}\left(x_{\mathbf{t}}^{0}\right)=0$, for all $\mathbf{t} \in E L$, and $\sum_{\mathbf{t} \in E L} u_{\mathbf{t}}\left(x_{\mathbf{t}}^{m_{\mathbf{t}}}\right)=1$

$u_{\mathbf{t}}\left(x_{\mathbf{t}}^{m_{\mathbf{t}}}\right) \geq u_{\mathbf{t}}\left(x_{\mathbf{t}}^{0}\right)$, for all $\mathbf{t} \in E L$,

$b_{h}^{\mathbf{s}} \geq b_{h-1}^{\mathbf{s}}+\varepsilon, h=1, \ldots, 5$, for all $\mathbf{s} \in\{\mathbf{1}, \ldots, \mathbf{6}\}$,

$b_{0}^{\mathbf{s}}=0$, and $b_{5}^{\mathbf{s}}=\sum_{\mathbf{t} \in E\left(G_{\mathbf{s}}\right)} u_{\mathbf{t}}\left(x_{\mathbf{t}}^{m_{\mathbf{t}}}\right)$, for all $\mathbf{s} \in\{\mathbf{1}, \ldots, \mathbf{6}\}$,

$b_{h}^{\mathbf{0}}=\sum_{\mathbf{s} \in\{\mathbf{1}, \ldots, \mathbf{6}\}} b_{h}^{\mathbf{s}}$, for all $h=0, \ldots, 5$. 
Table 3 Results after the first stage

\begin{tabular}{ll}
\hline Alternatives & {$\left[L_{\mathbf{0}}^{\mathcal{U}, P}, R_{\mathbf{0}}^{\mathcal{U}, P}\right]$} \\
\hline$A_{23}, A_{54}, A_{61}, A_{68}$ & {$\left[C_{1}, C_{4}\right]$} \\
$A_{36}, A_{80}, A_{81}$ & {$\left[C_{2}, C_{5}\right]$} \\
\hline
\end{tabular}

Table 4 Information provided by the expert in the second stage

\begin{tabular}{|c|c|c|c|c|c|}
\hline \multicolumn{2}{|c|}{ Capital adequacy } & \multicolumn{2}{|c|}{ Asset quality } & \multicolumn{2}{|c|}{ Management competence } \\
\hline Alternative & Assignment & Alternative & Assignment & Alternative & Assignment \\
\hline$A_{67}$ & {$\left[C_{1}, C_{2}\right]$} & $A_{60}$ & {$\left[C_{1}, C_{2}\right]$} & $A_{17}$ & $C_{2}$ \\
\hline$A_{19}$ & {$\left[C_{2}, C_{3}\right]$} & $A_{41}$ & {$\left[C_{2}, C_{3}\right]$} & $A_{60}$ & $C_{3}$ \\
\hline$A_{7}$ & $C_{3}$ & $A_{11}$ & {$\left[C_{3}, C_{4}\right]$} & $A_{1}$ & $C_{4}$ \\
\hline$A_{1}$ & $C_{4}$ & $A_{5}$ & {$\left[C_{4}, C_{5}\right]$} & & \\
\hline$A_{4}$ & $C_{5}$ & & & & \\
\hline Alternative & Assignment & Alternative & Assignment & Alternative & Assignment \\
\hline$A_{19}$ & $C_{1}$ & $A_{82}$ & $C_{1}$ & $A_{76}$ & {$\left[C_{1}, C_{2}\right]$} \\
\hline$A_{20}$ & $C_{2}$ & $A_{28}$ & $C_{2}$ & $A_{33}$ & $C_{3}$ \\
\hline$A_{3}$ & $C_{3}$ & $A_{79}$ & $C_{3}$ & $A_{22}$ & $C_{4}$ \\
\hline$A_{26}$ & $C_{4}$ & $A_{78}$ & {$\left[C_{3}, C_{4}\right]$} & $A_{47}$ & {$\left[C_{4}, C_{5}\right]$} \\
\hline$A_{36}$ & $C_{5}$ & $A_{55}$ & $C_{5}$ & & \\
\hline
\end{tabular}

Note that in this case we do not need the monotonicity constraint $u_{\mathbf{t}}\left(x_{\mathbf{t}}^{k}\right) \geq u_{\mathbf{t}}\left(x_{\mathbf{t}}^{k-1}\right), k=$ $1, \ldots, m_{\mathbf{t}}$, for any $\mathbf{t} \in E L$ because, as shown in Eq. (6), we are considering a linear marginal value function for each elementary criterion, and this function is defined by the marginal value $u_{\mathbf{t}}\left(x_{\mathbf{t}}\right)$ and by the worst and the best performances of the banks on each elementary criterion.

Solving the LP problem $\varepsilon^{*}=\max \varepsilon$, s.t. $E^{A^{R}}$, we find that $E^{A^{R}}$ is feasible and $\varepsilon^{*}>0$. This leads to the conclusion that there are multiple different instances of the preference model compatible with the above comprehensive judgments and preferential inputs. Clearly, the choice of a single decision instance from such limited information is likely to lead to conclusions that are not robust. Combining ROR with the modeling framework of the UTADIS method under the hierarchical structuring of the family of criteria, enables the formulation of results taking into account the full set of possible instances.

Applying (4), we computed the lowest and the highest possible class assignment for each alternative. Apart from the seven banks shown in Table 3, all the others could be possibly assigned to the whole range of classes. Moreover, applying (5), we computed the lowest and highest necessary assignment for each alternative. It appears that the set of necessary assignments is empty for all banks, since $L_{\mathbf{0}}^{\mathcal{U}, N}>R_{\mathbf{0}}^{\mathcal{U}, N}$ for all of them. It is evident that at this stage of the analysis, the obtained results are not conclusive enough.

In order to get a more clear recommendation, the expert analyst has to provide more detailed preference information. Then, the DM provided partial judgments involving the main CAMELS dimensions, as shown in Table 4. These partial judgments are easier for the DM to define, as each main dimension comprises a much smaller set of criteria compared to 
Table 5 Summary of possible assignments from the second stage of the analysis (non-reference alternatives)

\begin{tabular}{llllllll}
\hline Assignments & Overall & $\mathrm{CA}$ & $\mathrm{AS}$ & $\mathrm{MC}$ & $\mathrm{ER}$ & $\mathrm{LQ}$ & $\mathrm{SM}$ \\
\hline$C_{1}$ & - & - & 1 & - & - & - & - \\
{$\left[C_{1}, C_{2}\right]$} & - & - & 1 & - & 5 & 2 & 2 \\
{$\left[C_{1}, C_{3}\right]$} & - & - & - & 4 & 10 & 26 & 5 \\
{$\left[C_{1}, C_{4}\right]$} & 11 & - & 2 & 22 & 9 & 8 & 11 \\
{$\left[C_{1}, C_{5}\right]$} & 40 & - & 56 & 18 & 28 & 25 & - \\
$C_{2}$ & - & 2 & - & - & - & - & - \\
{$\left[C_{2}, C_{3}\right]$} & - & 13 & - & - & - & - & - \\
{$\left[C_{2}, C_{4}\right]$} & - & 2 & - & 7 & - & - & - \\
{$\left[C_{2}, C_{5}\right]$} & 16 & 28 & 17 & 24 & 15 & 19 & - \\
{$\left[C_{3}, C_{5}\right]$} & 3 & 34 & 4 & 7 & 9 & - & 33 \\
{$\left[C_{4}, C_{5}\right]$} & - & 3 & - & - & 3 & - & 30 \\
$C_{5}$ & - & - & - & - & 1 & - & - \\
Mean range & 4.5 & 3.1 & 4.6 & 4.0 & 3.9 & 3.9 & 2.7 \\
\hline & & & & & & & \\
\hline
\end{tabular}

the 31 criteria required for the comprehensive assignment decisions provided in the previous stage. The calculation of the new recommendation is performed analogously to the first stage.

With the new preference information, the integration of MCHP with the UTADIS ${ }^{G M S}$ method was employed again to get a new set of assignments. Table 5 reports the number of non-reference cases (i.e., banks-year observations not included in the assignments provided by the expert analyst), by the type of their assignment result (range of classes) at the comprehensive level and at all lower-level dimensions. In addition, the table also presents the mean range of the assignments as an indicator of the imprecision that describes the obtained results. The mean range is calculated from the number of classes in the sets of possible assignments, averaged over all non-reference bank-year observations.

It is evident that even with the new information, the sorting decisions at the comprehensive level are still characterized by ambiguity, as 40 (out of 70) cases can be assigned in any of the five rating classes. The examination of the partial assignments for each of the six main dimensions provides some insights on the decomposition of the banks' comprehensive performance and the sources of ambiguity in the assignments at the comprehensive level.

In particular, the partial assignments for capital adequacy (CA) and sensitivity to market risks (SM) are more precise compared to the other dimensions. In terms of capital adequacy, all banks are consistently rated in class $C_{2}$ or better (throughout the years), with 37 cases being in at least medium condition (i.e., belonging to categories $C_{3}-C_{5}$ ). This result is concordant with the characteristics of Greek banks during the period of the analysis, as prior to the outbreak of the Greek sovereign debt crisis in 2010, they have been generally well capitalized.

As far as their sensitivity to market risks is concerned, the banks also performed rather well over the period under consideration. In particular, 63 cases are considered as having at least medium performance on this dimension. There are, however, a few cases corresponding to banks that seem to be exposed to market risks (i.e., their assignment includes the high risk class $C_{1}$ ). These are mostly smaller banks, which have indeed developed some risky investment activities and financial products during that period.

Asset quality seems to be the main factor explaining the ambiguity in the assignment at the comprehensive level. In the majority of cases (56 out of 81), the assignments in this dimension span all five rating classes, which indicates that in order to obtain more precise 


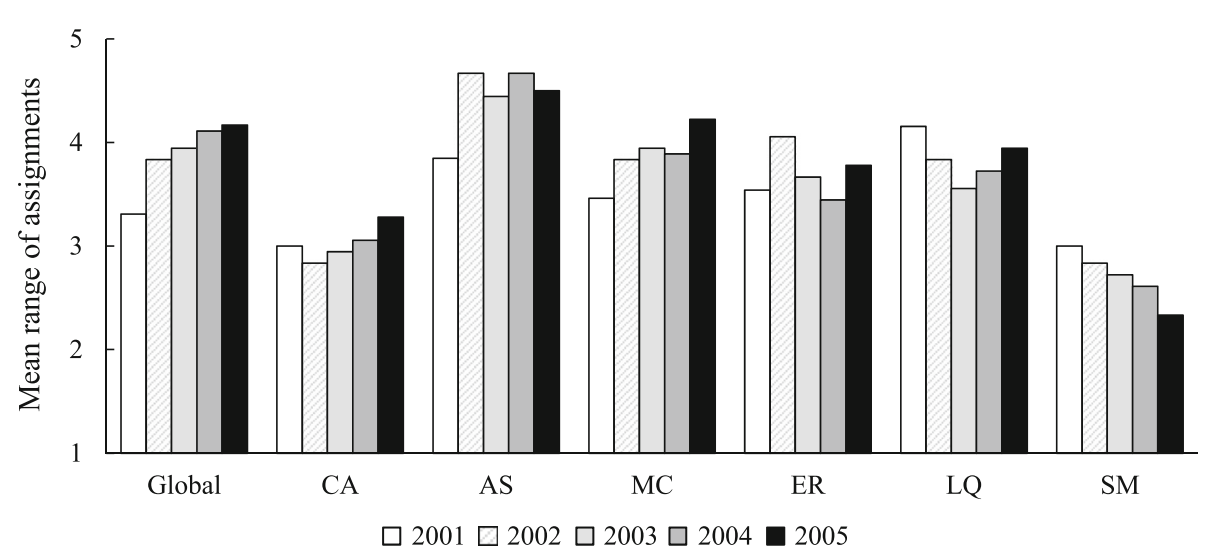

Fig. 2 Mean ranges of the assignments over time

Table 6 Summary of assignment results with the most discriminant value function (number of assignments by each class)

\begin{tabular}{lcrrrrrr}
\hline Class & Comprehensively & CA & AS & MC & ER & LQ & SM \\
\hline$C_{1}$ & 6 & 0 & 26 & 21 & 8 & 20 & 6 \\
$C_{2}$ & 23 & 22 & 9 & 29 & 24 & 38 & 4 \\
$C_{3}$ & 19 & 17 & 7 & 30 & 29 & 15 & 15 \\
$C_{4}$ & 32 & 43 & 7 & 5 & 15 & 8 & 42 \\
$C_{5}$ & 5 & 3 & 36 & 0 & 9 & 4 & 18 \\
\hline
\end{tabular}

conclusions on the asset quality dimension, further analysis is required using additional input information. The same applies (yet to a smaller extend) to management competence, earnings, and liquidity.

A further examination of the time trends in the range of the assignments over time (Fig. 2) reveals that the imprecision in the assignments at the comprehensive level has increased over time. This can be interpreted as a warning signal, as it implies that deriving clear conclusions on the overall performance of the banks became more difficult over the years. This trend was primarily driven by the increasing ambiguity in the evaluations with respect to capital adequacy (after 2002), asset quality (mostly in 2001-2002), and management competence. On the other hand, the imprecision in the evaluations with respect to the market risk dimension followed a declining trend, as the introduction of Greece to the Eurozone area in 2002 and the improving conditions in the global financial markets (particularly after 2003) contributed to the minimization of the exposure of Greek banks to external market risks.

The information derived from the imprecise assignments of the UTADIS ${ }^{G M S}$ method can be further enriched and complemented through the construction of the most discriminant additive value model, which is obtained through the solution of the optimization problem: $\max \varepsilon$, subject to $E^{A^{R}}$. Table 6 presents the number of assignments with the obtained model, both at the comprehensive level and at the level of the six performance dimensions. According to the results, there are six cases involving very high risk banks (class $C_{1}$ ), five cases of top performing banks (class $C_{5}$ ), whereas most banks are assigned to classes $C_{2}-$ $C_{5}$. The distribution of the assignments for the capital adequacy dimension resembles the assignment at the comprehensive level, whereas in terms of asset quality it is interesting to note that there is a considerable concentration in the two extreme rating classes. This 
Table 7 The relationship between the results of UTADIS ${ }^{G M S}$ and the assignments of the most discriminant model at the comprehensive level

\begin{tabular}{lllllll}
\hline & Mean value & \multicolumn{5}{l}{ Most discriminant assignments } \\
\cline { 3 - 7 } & & $C_{1}$ & $C_{2}$ & $C_{3}$ & $C_{4}$ & $C_{5}$ \\
\hline$\left[C_{1}, C_{4}\right]$ & 0.400 & $9.1 \%$ & $90.9 \%$ & - & - & - \\
{$\left[C_{1}, C_{5}\right]$} & 0.483 & $5.0 \%$ & $25.0 \%$ & $40.0 \%$ & $27.5 \%$ & $2.5 \%$ \\
{$\left[C_{2}, C_{5}\right]$} & 0.567 & - & - & - & $93.8 \%$ & $6.2 \%$ \\
{$\left[C_{3}, C_{5}\right]$} & 0.603 & - & - & - & $100.0 \%$ & - \\
& Mean value & 0.337 & 0.423 & 0.482 & 0.559 & 0.642 \\
\hline
\end{tabular}

is in accordance with the large number of imprecise assignments in this dimension, as discussed earlier. In terms of management competence and liquidity there is a concentration in classes $C_{1}-C_{3}$ (at most medium performance), whereas the results for market risk verify the remarks made earlier on the low exposure of Greek banks to external market risks as there is a clear concentration in classes $C_{4}-C_{5}$ (above average performance). The Kendall's $\tau$ rank correlations between the comprehensive assignment and the partial ones were higher for capital adequacy (0.725) and asset quality (0.653), which is concordant with the information that the expert analyst provided on the high importance of these criteria. The correlations of the comprehensive assignment to those of the other dimensions were lower (0.2-0.3).

Table 7 presents further results on the relationship of the imprecise assignments obtained by UTADIS ${ }^{G M S}$ with the ones of the most discriminant model at the comprehensive level. In particular, for banks assigned to different ranges of classes according to UTADIS ${ }^{G M S}$, we report their mean global values (i.e., performance scores) according to the most representative model (second column), as well as their distribution in the classes resulting from the most discriminant model (frequencies). For instance, the mean performance score for banks assigned to the range of classes $\left[C_{1}, C_{4}\right]$ is 0.4 , and most of such instances $(90.9 \%)$ are assigned to class $C_{2}$ by the most discriminant model. The last row in the table presents the mean comprehensive value for banks assigned to different classes by the most discriminant model. The results indicate that most banks assigned to $\left[C_{1}, C_{4}\right]$ by UTADIS ${ }^{G M S}$ are considered as low performance banks by the most discriminant model. Banks for which their assignment is completely imprecise according to UTADIS ${ }^{G M S}$ span the whole range of classes with the most discriminant model, but most of them are assigned to the medium performance class $C_{3}$. On the other hand, banks assigned to the range of classes $\left[C_{2}, C_{5}\right]$ and $\left[C_{3}, C_{5}\right]$ according to UTADIS ${ }^{G M S}$ are assigned to class $C_{4}$ by the most discriminant model. However, the mean value of banks in $\left[C_{2}, C_{5}\right]$ is 0.567 , which is very similar to the mean performance ( 0.559 ) of banks assigned to $C_{4}$ by the most discriminant model (i.e., they resemble typically good banks), whereas banks assigned to $\left[C_{3}, C_{5}\right]$ have a mean performance value of 0.603 , which is higher that the mean of class $C_{4}$ but lower that the mean of the top rating class $C_{5}(0.642)$.

\section{Conclusions}

Several methods are able to deal with multiple criteria sorting problems, but they all assume a single-level organization of the family of criteria. In this paper, we proposed an extension 
of the MCHP approach to sorting problems with a hierarchical structure of the family of criteria. The MCHP is a methodology that allows the decomposing of decision making problems into smaller dimensions (each taking into account different aspects of the problem). In this context, we introduced modeling formulations that allow the inference of a preference model from decision examples through preference disaggregation techniques based on an additive value function model (UTADIS and UTADIS ${ }^{G M S}$ methods). MCHP combined with UTADIS and UTADIS ${ }^{G M S}$ allows the consideration of both global and partial preference judgments, which adds flexibility to the specification of the input preference information required in the decision aiding process. The applicability of the MCHPbased methods was illustrated through an application regarding the assessment of bank performance.

Future research can be extended towards a number of different directions. First, similar approaches could be considered for other types of preference models for sorting problems, including outranking relation (Corrente et al. 2014), Choquet integral (Grabisch and Labreuche 2010), and rule-based models (Greco et al. 2010; Słowiński et al. 2000). That would be particularly useful, as it would yield a much more general MCHP framework, covering situations where different aspects of a decision problem require the adoption of different types of models. Group decision making problems can also be considered in such a context. Combinations with simulation methods (Kadziński and Tervonen 2013) could also be useful to enhance the assignment recommendations with probabilistic information, whereas further analysis could also focus on building good representative preference models in sorting problems with hierarchical structure, using the techniques presented in previous studies (Doumpos et al. 2014; Greco et al. 2011). In addition to these methodological extensions, further testing on other case studies and through experimental computational analyses could provide further insights into the properties of the MCHP-based sorting schemes. Introduction of procedures guiding the elicitation of preference information by the DM in the spirit of active learning would also be useful to reduce the cognitive effort required during the decision aiding process and make such techniques easier to apply in practice.

Acknowledgments This work has been partly funded by the "Programma Operativo Nazionale" Ricerca \& Competitivitá "2007-2013" within the project "PON04a2 E SINERGREEN-RES-NOVAE".

The fourth author wishes to acknowledge financial support from the Polish National Science Centre.

\section{Appendix}

\section{Proof of Proposition 3.1}

$(1) \Rightarrow(2)$ Let $a \underset{(\mathbf{r}, j)}{\longrightarrow}\left[C_{h_{j}}, C_{k_{j}}\right]$ for all $j=1, \ldots, n(\mathbf{r})$. This means that $b_{h_{j}-1}^{(\mathbf{r}, j)} \leq$ $U_{(\mathbf{r}, j)}(a)<b_{k_{j}}^{(\mathbf{r}, j)}$ for all $j=1, \ldots, n(\mathbf{r})$. Let us consider $h=\min _{j=1, \ldots, n(\mathbf{r})} h_{j}$ and $k=$ $\max _{j=1, \ldots, n(\mathbf{r})} k_{j}$. For the monotonicity of the thresholds, we shall have for all $j=1, \ldots, n(\mathbf{r})$ that $b_{h-1}^{(\mathbf{r}, j)} \leq b_{h_{j}-1}^{(\mathbf{r}, j)} \leq U_{(\mathbf{r}, j)}(a)<b_{k_{j}}^{(\mathbf{r}, j)} \leq b_{k}^{(\mathbf{r}, j)}$ for all $j=1, \ldots, n(\mathbf{r})$ and, consequently, $b_{h-1}^{(\mathbf{r}, j)} \leq U_{(\mathbf{r}, j)}(a)<b_{k}^{(\mathbf{r}, j)}$. Adding up with respect to $j$, we get

$$
b_{h-1}^{\mathbf{r}}=\sum_{j=1}^{n(\mathbf{r})} b_{h-1}^{(\mathbf{r}, j)} \leq \sum_{j=1}^{n(\mathbf{r})} U_{(\mathbf{r}, j)}(a)<\sum_{j=1}^{n(\mathbf{r})} b_{k}^{(\mathbf{r}, j)}=b_{k}^{\mathbf{r}} .
$$


From Eq. (1), it follows that $b_{h-1}^{\mathbf{r}} \leq U_{\mathbf{r}}(a)<b_{k}^{\mathbf{r}}$ and, consequently, $a \underset{\mathbf{r}}{\rightarrow}\left[C_{h}, C_{k}\right]$.

(2) $\Rightarrow$ (3) follows directly by setting $h_{j}=k_{j}=h$ for all $j=1, \ldots, n(\mathbf{r})$.

(3) $\Rightarrow$ (1) follows by contradiction, when we suppose that $b_{h}^{\mathbf{r}} \neq \sum_{j=1}^{n(\mathbf{r})} b_{h}^{(\mathbf{r}, j)}$ for some $h$. This implies that $b_{h}^{\mathbf{r}}>\sum_{j=1}^{n(\mathbf{r})} b_{h}^{(\mathbf{r}, j)}$ or $b_{h}^{\mathbf{r}}<\sum_{j=1}^{n(\mathbf{r})} b_{h}^{(\mathbf{r}, j)}$.

Let $b_{h}^{\mathbf{r}}>\sum_{j=1}^{n(\mathbf{r})} b_{h}^{(\mathbf{r}, j)}$ and $a \in A$ an alternative, such that

$$
U_{(\mathbf{r}, j)}(a)=b_{h}^{(\mathbf{r}, j)} \text { for all } j=1, \ldots, n(\mathbf{r}) .
$$

Obviously, this implies that $a \underset{(\mathbf{r}, j)}{\longrightarrow} C_{h+1}$ for all $j=1, \ldots, n(\mathbf{r})$. Adding up with respect to $j$ in the two members of Eq. (11), we get $U_{\mathbf{r}}(a)=\sum_{j=1}^{n(\mathbf{r})} U_{(\mathbf{r}, j)}(a)=\sum_{j=1}^{n(\mathbf{r})} b_{h}^{(\mathbf{r}, j)}<b_{h}^{\mathbf{r}}$ and, consequently, $a \underset{\mathbf{r}}{\rightarrow} C_{\leq h}$, being in contradiction with the hypothesis.

Now, let $b_{h}^{\mathbf{r}}<\sum_{j=1}^{n(\mathbf{r})} b_{h}^{(\mathbf{r}, j)}$ and $a \in A$ an alternative, such that

$$
U_{(\mathbf{r}, j)}(a)=b_{h}^{(\mathbf{r}, j)}-\frac{\varepsilon}{n(\mathbf{r})} \text { for all } j=1, \ldots, n(\mathbf{r})
$$

where $\varepsilon>0$. This choice implies that $a \underset{(\mathbf{r}, \mathbf{j})}{\longrightarrow} C_{\leq h}$, for all $j=1, \ldots, n(\mathbf{r})$. Now, adding up with respect to $j$ in the two members of Eq. (12), we get $U_{\mathbf{r}}(a)=\sum_{j=1}^{n(\mathbf{r})} U_{(\mathbf{r}, j)}(a)=$ $\sum_{j=1}^{n(\mathbf{r})}\left[b_{h}^{(\mathbf{r}, j)}-\frac{\varepsilon}{n(\mathbf{r})}\right]=\sum_{j=1}^{n(\mathbf{r})} b_{h}^{(\mathbf{r}, j)}-\varepsilon$. If we choose $\varepsilon$ such that

$$
0<\varepsilon \leq \min \left\{\min \left\{n(\mathbf{r}) \cdot\left[b_{h}^{(\mathbf{r}, j)}-b_{h-1}^{(\mathbf{r}, j)}\right], j=1, \ldots, n(\mathbf{r})\right\}, \sum_{j=1}^{n(\mathbf{r})} b_{h}^{(\mathbf{r}, j)}-b_{h}^{\mathbf{r}}\right\}
$$

we obtain that $b_{h-1}^{(\mathbf{r}, j)} \leq U_{(\mathbf{r}, j)}(a)<b_{h}^{(\mathbf{r}, j)}$ for all $j=1, \ldots, n(\mathbf{r})^{2}$ and $U_{\mathbf{r}}(a)>b_{h}^{\mathbf{r} 3}$ implying that $a \underset{(\mathbf{r}, j)}{\longrightarrow} C_{h}$ for all $j=1, \ldots, n(\mathbf{r})$ and $a \underset{\mathbf{r}}{\rightarrow} C_{\geq h+1}$, thus leading to a contradiction.

\section{Proof of Proposition 4.1}

1. Let $a \in A, \mathbf{r} \in \mathcal{I}_{\mathcal{G}} \backslash E L$ and $h=2, \ldots, p$ such that $\operatorname{not}\left(a \underset{\mathbf{r}}{\stackrel{N}{\longrightarrow}} C_{\geq h}\right)$. This means that there exists at least one $(U, b)$ such that $U_{\mathbf{r}}(a)<b_{h-1}^{\mathbf{r}}$. Therefore $a \stackrel{P}{\rightarrow} C_{\leq h-1}$. Let us observe that $a \stackrel{N}{\underset{\mathbf{r}}{\longrightarrow}} C_{\geq h}$ and $a \underset{\mathbf{r}}{\stackrel{P}{\rightarrow}} C_{\leq h-1}$ do not hold simultaneously because, otherwise, a couple $(\bar{U}, \bar{b})$ should exist, such that $\bar{U}_{\mathbf{r}}(a) \geq \bar{b}_{h-1}^{\mathbf{r}}$ and $\bar{U}_{\mathbf{r}}(a)<\bar{b}_{h-1}^{\mathbf{r}}$, which is impossible.

2. Let $a \in A, \mathbf{r} \in \mathcal{I}_{\mathcal{G}} \backslash E L$ and $k=1, \ldots, p-1$ such that $\operatorname{not}\left(a \stackrel{N}{\rightarrow} C_{\leq k}\right)$. This means that there exists at least one $(U, b)$ such that $U_{\mathbf{r}}(a) \geq b_{k}^{\mathbf{r}}$. Therefore $a \underset{\mathbf{r}}{\stackrel{P}{\rightarrow}} C_{\geq k+1}$. Let us observe that $a \underset{\mathbf{r}}{\stackrel{N}{\longrightarrow}} C_{\leq k}$ and $a \underset{\mathbf{r}}{\stackrel{P}{\longrightarrow}} C_{\geq k+1}$ do not hold simultaneously because, otherwise, a couple $(\bar{U}, \bar{b})$ should exist, such that $\bar{U}_{\mathbf{r}}(a)<\bar{b}_{k}^{\mathbf{r}}$ and $\bar{U}_{\mathbf{r}}(a) \geq \bar{b}_{k}^{\mathbf{r}}$, which is impossible.

\footnotetext{
2 Because $\varepsilon \leq \min _{j=1, \ldots, n(\mathbf{r})} n(\mathbf{r})\left[b_{h}^{(\mathbf{r}, j)}-b_{h-1}^{(\mathbf{r}, j)}\right]$.

3 Because $\varepsilon \leq \sum_{j=1}^{n(\mathbf{r})} b_{h}^{(\mathbf{r}, j)}-b_{h}^{\mathbf{r}}$ and, consequently $\sum_{j=1}^{n(\mathbf{r})} b_{h}^{(\mathbf{r}, j)}-\varepsilon>b_{h}^{\mathbf{r}}$.
} 
3. $a \underset{(\mathbf{r}, j)}{\stackrel{N}{\longrightarrow}} C_{\geq h_{j}}$ for all $j=1, \ldots, n(\mathbf{r})$ implies that $U_{(\mathbf{r}, j)}(a) \geq b_{h_{j}-1}^{(\mathbf{r}, j)}$ for all $(U, b)$ and for all $j=1, \ldots, n(\mathbf{r})$. Considering $h=\min _{j=1, \ldots, n(\mathbf{r})} h_{j}$, for the monotonicity of the thresholds we have that $U_{(\mathbf{r}, j)}(a) \geq b_{h-1}^{(\mathbf{r}, j)}$ for all $(U, b)$ and for all $j$. As a consequence, adding up with respect to $j$, we get $U_{\mathbf{r}}(a)=\sum_{j=1}^{n(\mathbf{r})} U_{(\mathbf{r}, j)}(a) \geq \sum_{j=1}^{n(\mathbf{r})} b_{h-1}^{(\mathbf{r}, j)}=b_{h-1}^{\mathbf{r}}$ for all $(U, b)$, which proves point 2 .

4. $a \underset{(\mathbf{r}, j)}{\stackrel{N}{\longrightarrow}} C_{\leq k_{j}}$ for all $j=1, \ldots, n(\mathbf{r})$ implies that $U_{(\mathbf{r}, j)}(a)<b_{k_{j}}^{(\mathbf{r}, j)}$ for all $(U, b)$ and for all $j=1, \ldots, n(\mathbf{r})$. Considering $k=\max _{j=1, \ldots, n(\mathbf{r})} k_{j}$, for the monotonicity of the thresholds we have that $U_{(\mathbf{r}, j)}(a)<b_{k}^{(\mathbf{r}, j)}$ for all $(U, b)$ and for all $j$. As a consequence, adding up with respect to $j$, we get $U_{\mathbf{r}}(a)=\sum_{j=1}^{n(\mathbf{r})} U_{(\mathbf{r}, j)}(a)<\sum_{j=1}^{n(\mathbf{r})} b_{k}^{(\mathbf{r}, j)}=b_{k}^{\mathbf{r}}$ for all $(U, b)$, which implies point 3 .

5. $a \underset{(\mathbf{r}, j)}{\stackrel{N}{\longrightarrow}} C_{\geq h_{j}}$, for all $j \in\{1, \ldots, n(\mathbf{r})\} \backslash\{\bar{j}\}$ implies that for all $(U, b), U_{(\mathbf{r}, j)}(a) \geq$ $b_{h_{j}-1}^{(\mathbf{r}, j)}$ for all $j \in\{1, \ldots, n(\mathbf{r})\} \backslash\{\bar{j}\}$. Analogously, $a \underset{(\mathbf{r}, \bar{j})}{\stackrel{P}{\longrightarrow}} C_{\geq h_{\bar{j}}}$ implies that there exists at least one $(\bar{U}, \bar{b})$ such that $\bar{U}_{(\mathbf{r}, \bar{j})}(a) \geq \bar{b}_{h_{\bar{j}}-1}^{(\mathbf{r}, \bar{j})}$. Considering $h=\min _{j=1, \ldots, n(\mathbf{r})} h_{j}$, for $(\bar{U}, \bar{b})$ and for the monotonicity of the thresholds we have that $\bar{U}_{(\mathbf{r}, j)}(a) \geq \bar{b}_{h-1}^{(\mathbf{r}, j)}$ for all $j=1, \ldots, n(\mathbf{r})$. Adding up with respect to $j$ we get $\bar{U}_{\mathbf{r}}(a)=\sum_{j=1}^{n(\mathbf{r})} \bar{U}_{(\mathbf{r}, j)}(a) \geq$ $\sum_{j=1}^{n(\mathbf{r})} \bar{b}_{h-1}^{(\mathbf{r}, j)}=\bar{b}_{h-1}^{\mathbf{r}}$, which proves point 4 .

6. $a \underset{(\mathbf{r}, j)}{\stackrel{N}{\longrightarrow}} C_{\leq k_{j}}$, for all $j \in\{1, \ldots, n(\mathbf{r})\} \backslash\{\bar{j}\}$ implies that for all $(U, b), U_{(\mathbf{r}, j)}(a)<b_{k_{j}}^{(\mathbf{r}, j)}$ for all $j \in\{1, \ldots, n(\mathbf{r})\} \backslash\{\bar{j}\}$. Analogously, $a \underset{(\mathbf{r}, \bar{j})}{\stackrel{P}{\longrightarrow}} C_{\leq k_{\bar{j}}}$ implies that there exists at least one $(\bar{U}, \bar{b})$ such that $\bar{U}_{(\mathbf{r}, \bar{j})}(a)<\bar{b}_{k_{\bar{j}}}^{(\mathbf{r}, \bar{j})}$. Considering $k=\max _{j=1, \ldots, n(\mathbf{r})} k_{j}$, for $(\bar{U}, \bar{b})$ and for the monotonicity of the thresholds we have that $\bar{U}_{(\mathbf{r}, j)}(a)<\bar{b}_{k}^{(\mathbf{r}, j)}$ for all $j=1, \ldots, n(\mathbf{r})$. Adding up with respect to $j$ we get $\bar{U}_{\mathbf{r}}(a)=\sum_{j=1}^{n(\mathbf{r})} \bar{U}_{(\mathbf{r}, j)}(a)<$ $\sum_{j=1}^{n(\mathbf{r})} \bar{b}_{k}^{(\mathbf{r}, j)}=\bar{b}_{k}^{\mathbf{r}}$, which proves point 5 .

\section{Proof of Proposition 4.2}

1. Let $L_{(\mathbf{r}, j)}^{\mathcal{U}, P}(a)=h_{j}$ for all $j=1, \ldots, n(\mathbf{r})$. This means that $a \underset{(\mathbf{r}, j)}{\stackrel{N}{\longrightarrow}} C_{\geq h_{j}}$ and $\operatorname{not}\left(a \underset{(\mathbf{r}, j)}{\stackrel{N}{\longrightarrow}} C_{\geq l}\right)$ with $l>h_{j}$ for all $j=1, \ldots, n(\mathbf{r})$. By Proposition 4.1 we get $a \underset{\mathbf{r}}{\stackrel{N}{\rightarrow}} C_{\geq h}$ with $h=\min _{j=1, \ldots, n(\mathbf{r})} h_{j}$. As a consequence we get the thesis.

2. Let $R_{(\mathbf{r}, j)}^{\mathcal{U}, P}(a)=k_{j}$ for all $j=1, \ldots, n(\mathbf{r})$. This means that $a \underset{(\mathbf{r}, j)}{\stackrel{N}{\longrightarrow}} C_{\leq k_{j}}$ and $\operatorname{not}\left(a \underset{(\mathbf{r}, j)}{\stackrel{N}{\longrightarrow}} C_{\geq l}\right)$ with $l>k_{j}$ for all $j=1, \ldots, n(\mathbf{r})$. By Proposition 4.1 we get $a \underset{\mathrm{r}}{\stackrel{N}{\longrightarrow}} C_{\geq k}$ with $k=\max _{j=1, \ldots, n(\mathbf{r})} k_{j}$. As a consequence we get the thesis. 


\section{References}

Angilella, S., Corrente, S., Greco, S., \& Słowiński, R. (2013). Multiple criteria hierarchy process for the Choquet integral. In R. C. Purshouse, P. J. Fleming, C. M. Fonseca, S. Greco, \& J. Shaw, (Eds.), Evolutionary multi-criterion optimization, volume 7811 of lecture notes in computer science (pp. 475-489). Berlin: Springer.

Bana e Costa, C. A., \& Jean-Claude Vansnick,. (2008). A critical analysis of the eigenvalue method used to derive priorities in AHP. European Journal of Operational Research, 187(3), 1422-1428.

Bouyssou, D., \& Marchant, T. (2010). Additive conjoint measurement with ordered categories. European Journal of Operational Research, 203(1), 195-204.

Comptroller of the Currency Administrator of National Banks. Bank supervision process. Available at: http:// www.occ.gov/publications/publications-by-type/comptrollers-handbook/banksupervisionprocess.html, 2007

Corrente, S., Greco, S., Kadziński, M., \& Słowiński, R. (2014). Robust ordinal regression. Wiley Encyclopedia of Operations Research and Management Science (pp. 1-10).

Corrente, S., Figueira, J. R., \& Greco, S. (2014). Dealing with interaction between bipolar multiple criteria preferences in PROMETHEE methods. Annals of Operations Research, 217, 137-164.

Corrente, S., Greco, S., Kadziński, M., \& Słowiński, R. (2013). Robust ordinal regression in preference learning and ranking. Machine Learning, 93, 381-422.

Corrente, S., Greco, S., \& Słowiński, R. (2012). Multiple criteria hierarchy process in robust ordinal regression. Decision Support Systems, 53(3), 660-674.

Corrente, S., Greco, S., \& Słowiński, R. (2013). Multiple criteria hierarchy process with ELECTRE and PROMETHEE. Omega, 41, 820-846.

Devaud, J. M., Groussaud, G., \& Jacquet-Lagréze, E. (1980). Une méthode de construction de fonctions d'utilité additives rendant compte de judgments globaux. In Proceedings of EURO Working Group Meeting on Multicriteria Decision Aiding, Bochum.

Doumpos, M., \& Zopounidis, C. (2002). Multicriteria decision aid classification methods. New York: Springer.

Doumpos, M., \& Zopounidis, C. (2010). A multicriteria decision support system for bank rating. Decision Support Systems, 50(1), 55-63.

Doumpos, M., Zopounidis, C., \& Galariotis, E. (2014). Inferring robust decision models in multicriteria classification problems: An experimental analysis. European Journal of Operational Research, 236, 601-611.

Grabisch, M., \& Labreuche, C. (2010). A decade of application of the Choquet and Sugeno integrals in multi-criteria decision aid. Annals of Operations Research, 1(175), 247-286.

Greco, S., Kadziński, M., \& Słowiński, R. (2011). Selection of a representative value function in robust multiple criteria sorting. Computers \& Operations Research, 38, 1620-1637.

Greco, S., Matarazzo, B., \& Słowiński, R. (1999). The use of rough sets and fuzzy sets in MCDM. In T. Gal, T. Stewart, \& T. Hanne, (Eds.), Advances in multiple criteria decision making, chapter 14 (pp. 14.1-14.59). Dordrecht: Kluwer Academic.

Greco, S., Matarazzo, B., \& Słowiński, R. (2001). Rough sets theory for multicriteria decision analysis. European Journal of Operational Research, 129(1), 1-47.

Greco, S., Matarazzo, B., \& Słowiński, R. (2002). Rough sets methodology for sorting problems in presence of multiple attributes and criteria. European Journal of Operational Research, 138(2), 247-259.

Greco, S., Matarazzo, B., \& Słowiński, R. (2010). Dominance-based rough set approach to decision under uncertainty and time preference. Annals of Operations Research, 176(1), 41-75.

Greco, S., Mousseau, V., \& Słowiński, R. (2008). Ordinal regression revisited: multiple criteria ranking using a set of additive value functions. European Journal of Operational Research, 191(2), 416-436.

Greco, S., Mousseau, V., \& Słowiński, R. (2010). Multiple criteria sorting with a set of additive value functions. European Journal of Operational Research, 207(3), 1455-1470.

Jacquet-Lagrèze, E., \& Siskos, Y. (2001). Preference disaggregation: 20 years of MCDA experience. European Journal of Operational Research, 130(2), 233-245.

Kadziński, M., \& Tervonen, T. (2013). Stochastic ordinal regression for multiple criteria sorting problems. Decision Support Systems, 55(11), 55-66.

Keeney, R. L., \& Raiffa, H. (1993). Decisions with multiple objectives: Preferences and value tradeoffs. New York: Wiley.

Saaty, T. L. (2005). The analytic hierarchy and analytic network processes for the measurement of intangible criteria and for decision-making. In J. Figueira, S. Greco, \& M. Ehrgott (Eds.), Multiple criteria decision analysis: State of the art surveys (pp. 345-382). Berlin: Springer.

Sahajwala, R., \& Van den Bergh, P. (December 2000). Supervisory risk assessment and early warning systems. Technical Report 4, Bank of International Settlements, Basel. 
Słowiński, R., Greco, S., \& Matarazzo, B. (2002). Rough set analysis of preference-ordered data. In J. J Alpigini, J. F. Peters, A. Skowron, \& N. Zhong, (Eds.), Rough sets and current trends in computing, volume 2475 of lecture notes in artificial intelligence (pp. 44-59). Berlin: Springer.

Słowiński, R., Stefanowski, J., Greco, S., \& Matarazzo, B. (2000). Rough set based processing of inconsistent information in decision analysis. Control and Cybernetics, 29, 379-404.

van Greuning, H., \& Brajovic Bratanovic, S. (2009). Analyzing banking risk-a framework for assessing corporate governance and risk management (3rd ed.). Washington, DC: The World Bank.

Wakker, P. P. (1989). Additive representations of preferences: A new foundation of decision analysis, volume 4 of Theory and Decision Library $C$. Berlin: Springer.

Yu, W. (1992). Aide multicritère à la décision dans le cadre de la problématique du tri: méthodes et applications. Ph.D. thesis, LAMSADE, Université Paris Dauphine, Paris.

Zopounidis, C., \& Doumpos, M. (1999). A multicriteria decision aid methodology for sorting decision problems: The case of financial distress. Computational Economics, 14, 197-218.

Zopounidis, C., \& Doumpos, M. (2002). Multicriteria classification and sorting methods: A literature review. European Journal of Operational Research, 138, 229-246. 